\begin{tabular}{|c|c|}
\hline Title & Lightcone dual ities for curves in the lightcone unit 3-sphere \\
\hline Author(s) & Izumiya, Shy uichi; Jiang, Y ang; Sato, T akami \\
\hline Citation & $\begin{array}{l}\text { Journal of Mathematical Physics, 54(6), } 063511 \\
\text { https://doi.org/10.1063/1.4811451 }\end{array}$ \\
\hline Issue Date & $2013-06$ \\
\hline Doc URL & http:/hdl.handle.net/2115/53140 \\
\hline Rights & $\begin{array}{l}\text { Copyright } 2013 \text { A merican Institute of Phy sics. This article may be downloaded for personal use only. A ny other use } \\
\text { requires prior permission of the author and the A merican Institute of Physics. The following article appeared in J. Math. } \\
\text { Phys. } 54,063511 \text { (2013) and may be found at http://mp.aip.org/resource } / \not \text { mapaq } / 5416 \text { po63511_s1. }\end{array}$ \\
\hline Type & article \\
\hline File Information & JMathPhys_54_063511.pdf \\
\hline
\end{tabular}

Instructions for use 


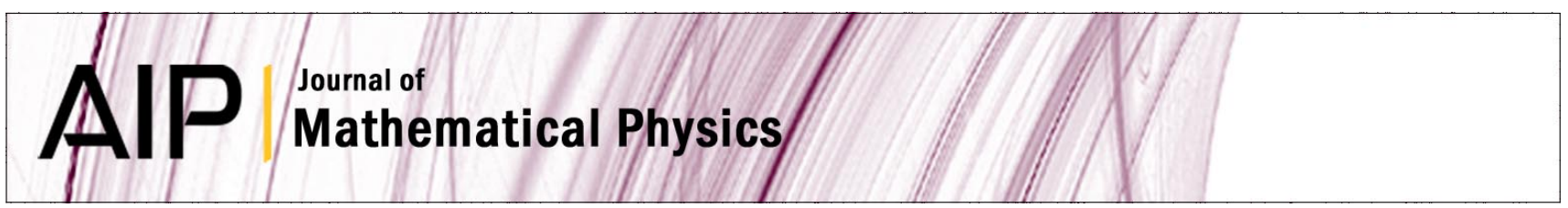

\section{Lightcone dualities for curves in the lightcone unit 3-sphere}

Shyuichi Izumiya, Yang Jiang, and Takami Sato

Citation: J. Math. Phys. 54, 063511 (2013); doi: 10.1063/1.4811451

View online: http://dx.doi.org/10.1063/1.4811451

View Table of Contents: http://jmp.aip.org/resource/1/JMAPAQ/v54/i6

Published by the AIP Publishing LLC.

\section{Additional information on J. Math. Phys.}

Journal Homepage: http://jmp.aip.org/

Journal Information: http://jmp.aip.org/about/about_the_journal

Top downloads: http://jmp.aip.org/features/most_downloaded

Information for Authors: http://jmp.aip.org/authors

\section{ADVERTISEMENT}

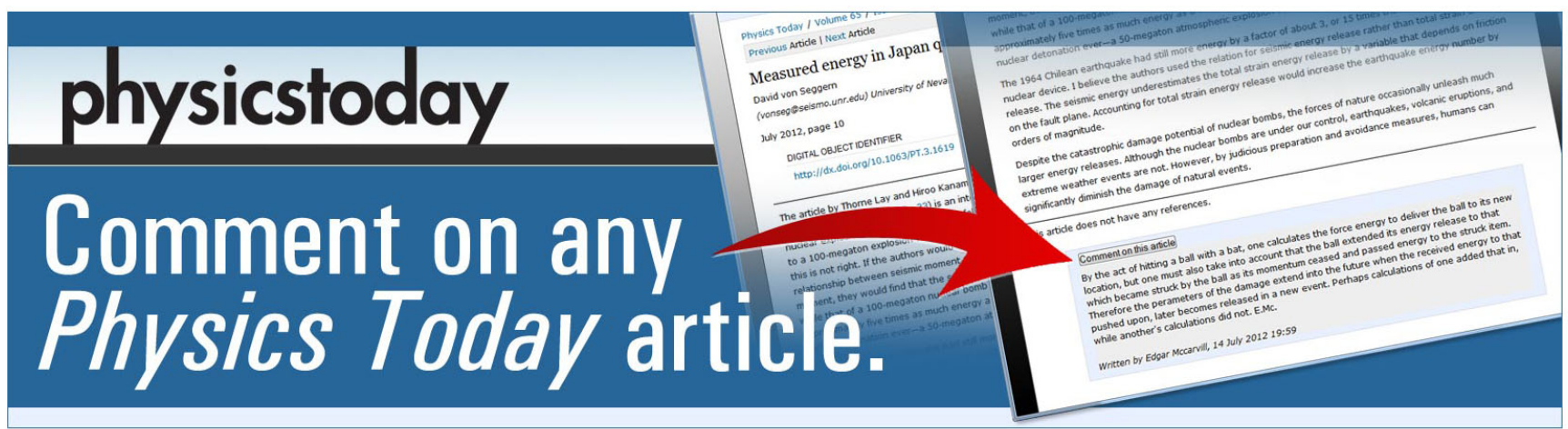




\title{
Lightcone dualities for curves in the lightcone unit 3-sphere
}

\author{
Shyuichi Izumiya, ${ }^{1, a)}$ Yang Jiang, ${ }^{1,2, b)}$ and Takami Sato ${ }^{2, c)}$ \\ ${ }^{1}$ School of Mathematics and Statistics, Northeast Normal University, Changchun 130024, \\ People's Republic of China \\ ${ }^{2}$ Department of Mathematics, Hokkaido University, Sapporo 060-0810, Japan
}

(Received 25 December 2012; accepted 4 June 2013; published online 20 June 2013)

\begin{abstract}
In this paper, we consider the curves in the unit 3-sphere in the lightcone. The unit 3-sphere can be canonically embedded in the lightcone and de Sitter 4-space in Lorentz-Minkowski 5-space. We investigate these curves in the framework of the theory of Legendrian dualities between pseudo-spheres in Lorentz-Minkowski 5-space. () 2013 AIP Publishing LLC. [http://dx.doi.org/10.1063/1.4811451]
\end{abstract}

\section{INTRODUCTION}

The evolutes of curves in the unit 2-sphere are studied in Ref. 7 from the view point of the Legendrian duality. ${ }^{4,5}$ It is known that the evolute of a curve in the unit 2-sphere is the dual of the tangent indicatrix of the original curve. ${ }^{11}$ The dual curve in the unit 2-sphere is defined to be equidistant by $\pi / 2$ from the original one. For a curve in the unit 3 -sphere, however, the dual is a surface. Therefore, the dual of the tangent indicatrix of a curve is a surface which is called the focal surface (or, the focal set) of the original curve. The critical locus of the focal surface is the evolute of the original curve (cf. Ref. 11). We remark that the focal set of a curve in the unit 2-sphere is a curve which is equal to the evolute.

On the other hand, the first author (S.I.) introduced the mandala of Legendrian dualities between pseudo-spheres in Lorentz-Minkowski 5-space., ${ }^{4,5}$ There are three kinds of pseudo-spheres in Lorentz-Minkowski 5-space (i.e., the hyperbolic space, the de Sitter space, and the lightcone). Especially, if we investigate spacelike submanifolds in the lightcone, those Legendrian dualities are essentially useful (see also Ref. 9). For the de Sitter 4-space and the lightcone in Lorentz-Minkowski 5 -space, there exist naturally embedded unit 3-spheres. The de Sitter 4-space corresponds to the cosmic model, and the lightcone also has its clear background in Physics. ${ }^{15}$ In this paper, we investigate the curves in the unit 3-sphere in the framework of the theory of Legendrian dualities between pseudo-spheres in Lorentz-Minkowski 5-space (Refs. 1, 2, 5, 6, 10-13, etc.). If we have a regular curve in the unit 3-sphere, then we have the regular curve in the embedded unit 3-sphere in the lightcone or de Sitter space. Therefore, we naturally have the dual hypersurfaces in the lightcone as an application of the duality theorem in Ref. 5. There are two kinds of lightcone dual hypersurfaces of a curve in the unit 3-sphere. We will give the classifications of the singularities of these hypersurfaces. In physics, the singularities of the lightcone are also studied. ${ }^{14}$ The critical value sets of these two hypersurfaces are called the lightcone focal surfaces, respectively. The projections of these focal surfaces to unit 3-sphere are different surfaces. In Ref. 7, we have shown that the projection images of the critical value sets of lightcone dual hypersurfaces for a curve in the unit 2-sphere coincide with the evolute of the original curve. Therefore, the situation of curves in the unit 3 -sphere is quite different from that of curves in the unit 2-sphere. However, the projections of the critical sets of lightcone focal surfaces are equal to the evolute of the curve. In order to clarify such situation, we

\footnotetext{
a) Present address: Department of Mathematics, Hokkaido University, Sapporo 060-0810, Japan. Electronic mail: izumiya@math.sci.hokudai.ac.jp.

b)E-mail: xjiangyang@126.com

c)E-mail: taka_mi@ec.hokudai.ac.jp
} 
introduce the notion of discriminant set of higher order for unfoldings of functions of one-variable (see Sec. VI).

A brief description of the organization for this paper is as follows: In Sec. II, we give basic concepts in this paper. In Sec. III, we formulate the Frenet-Serret type formulae for the curves in the unit 3-sphere. We also give the definition of the spherical focal surfaces of the curve in the unit 3-sphere. The spherical evolutes of the curve in the unit 3-sphere are given by the critical sets of the spherical focal surfaces. In Sec. IV, we define the lightcone dual hypersurfaces and the ligtcone focal surfaces of the curves in the unit 3-sphere. In Sec. V, we calculate the conditions for the $A_{k}(k$ $=1,2,3,4)$ singularities for the lightcone height functions of the curves in the unit 3-sphere. In Sec. VI, we show that the projections of the critical sets of focal surfaces to the unit 3-sphere are the same, and they are equal to the spherical evolutes of the curve in the unit 3-sphere (Theorem 6.2). We also study the singularities of the lightcone dual hypersurfaces, the lightcone focal surfaces and the spherical evolutes of the curves in the unit 3-sphere which is one of the main results in this paper (cf. Theorem 6.9).

All maps considered here are of class $C^{\infty}$ unless otherwise stated.

\section{BASIC CONCEPTS}

In this section, we give the basic concepts in this paper. Let $\mathbb{R}^{5}$ be a five-dimensional vector space. For any two vectors $\boldsymbol{x}=\left(x_{0}, x_{1}, x_{2}, x_{3}, x_{4}\right), \boldsymbol{y}=\left(y_{0}, y_{1}, y_{2}, y_{3}, y_{4}\right)$ in $\mathbb{R}^{5}$, their pseudo-scalar product is defined by $\langle\boldsymbol{x}, \boldsymbol{y}\rangle=-x_{0} y_{0}+x_{1} y_{1}+x_{2} y_{2}+x_{3} y_{3}+x_{4} y_{4}$. The pair $\left(\mathbb{R}^{5},\langle\rangle,\right)$ is called Lorentz-Minkowski 5-space. We denote it as $\mathbb{R}_{1}^{5}$.

For any four vectors $\boldsymbol{x}=\left(x_{0}, x_{1}, x_{2}, x_{3}, x_{4}\right), \boldsymbol{y}=\left(y_{0}, y_{1}, y_{2}, y_{3}, y_{4}\right), \boldsymbol{z}=\left(z_{0}, z_{1}, z_{2}, z_{3}, z_{4}\right)$, $\boldsymbol{w}=\left(w_{0}, w_{1}, w_{2}, w_{3}, w_{4}\right) \in \mathbb{R}_{1}^{5}$, their pseudo-vector product is defined by

$$
\boldsymbol{x} \wedge \boldsymbol{y} \wedge \boldsymbol{z} \wedge \boldsymbol{w}=\left|\begin{array}{ccccc}
-\boldsymbol{e}_{0} & \boldsymbol{e}_{1} & \boldsymbol{e}_{2} & \boldsymbol{e}_{3} & \boldsymbol{e}_{4} \\
x_{0} & x_{1} & x_{2} & x_{3} & x_{4} \\
y_{0} & y_{1} & y_{2} & y_{3} & y_{4} \\
z_{0} & z_{1} & z_{2} & z_{3} & z_{4} \\
w_{0} & w_{1} & w_{2} & w_{3} & w_{4}
\end{array}\right|,
$$

where $\left\{\boldsymbol{e}_{0}, \boldsymbol{e}_{1}, \boldsymbol{e}_{2}, \boldsymbol{e}_{3}, \boldsymbol{e}_{4}\right\}$ is the canonical basis of $\mathbb{R}_{1}^{5}$. A non-zero vector $\boldsymbol{x} \in \mathbb{R}_{1}^{5}$ is called spacelike, lightlike, or timelike if $\langle\boldsymbol{x}, \boldsymbol{x}\rangle>0,\langle\boldsymbol{x}, \boldsymbol{x}\rangle=0$, or $\langle\boldsymbol{x}, \boldsymbol{x}\rangle<0$, respectively. The norm of $\boldsymbol{x} \in \mathbb{R}_{1}^{5}$ is defined by $\|\boldsymbol{x}\|=(\operatorname{sign}(\boldsymbol{x})\langle\boldsymbol{x}, \boldsymbol{x}\rangle)^{1 / 2}$, where $\operatorname{sign}(\boldsymbol{x})$ denotes the signature of $\boldsymbol{x}$ which is given by $\operatorname{sign}(\boldsymbol{x})=1,0$, or -1 when $\boldsymbol{x}$ is a spacelike, lightlike, or timelike vector, respectively.

Let $\boldsymbol{\gamma}: I \rightarrow \mathbb{R}_{1}^{5}$ be a regular curve in $\mathbb{R}_{1}^{5}$ (i.e., $\dot{\gamma}(t) \neq \mathbf{0}$ for any $t \in I$ ), where $I$ is an open interval. For any $t \in I$, the curve $\boldsymbol{\gamma}$ is called spacelike, lightlike, or timelike if $\langle\dot{\gamma}(t), \dot{\gamma}(t)\rangle>0,\langle\dot{\gamma}(t), \dot{\gamma}(t)\rangle=0$, or $\langle\dot{\gamma}(t), \dot{\gamma}(t)\rangle<0$, respectively. The arc-length of a spacelike or timelike curve $\boldsymbol{\gamma}$ measured from $\boldsymbol{\gamma}\left(t_{0}\right)\left(t_{0} \in I\right)$ is $s(t)=\int_{t_{0}}^{t}\|\dot{\gamma}(t)\| \mathrm{d} t$.

The parameter $s$ is determined such that $\left\|\boldsymbol{\gamma}^{\prime}(s)\right\|=1$ for the nonlightlike curve, where $\boldsymbol{\gamma}^{\prime}(s)$ $=\mathrm{d} \boldsymbol{\gamma} / \mathrm{d} s(s)$ is the unit tangent vector of $\boldsymbol{\gamma}$ at $s$. We define the de Sitter 4-spaceby

$$
S_{1}^{4}=\left\{\boldsymbol{x} \in \mathbb{R}_{1}^{5} \mid\langle\boldsymbol{x}, \boldsymbol{x}\rangle=1\right\}
$$

We define the closed lightcone with the vertex $\boldsymbol{a}$ by

$$
L C_{a}=\left\{\boldsymbol{x} \in \mathbb{R}_{1}^{5} \mid\langle\boldsymbol{x}-\boldsymbol{a}, \boldsymbol{x}-\boldsymbol{a}\rangle=0\right\} .
$$

We define the open lightcone at the origin by

$$
L C^{*}=\left\{\boldsymbol{x} \in \mathbb{R}_{1}^{5} \backslash\{\boldsymbol{0}\} \mid\langle\boldsymbol{x}, \boldsymbol{x}\rangle=0\right\} .
$$

Here we consider the unit sphere in the lightcone defined by

$$
S_{+}^{3}=\left\{\boldsymbol{x} \in L C^{*} \mid x_{0}=1\right\}=\left\{\boldsymbol{x} \in \mathbb{R}_{1}^{5} \mid x_{0}=1, x_{1}^{2}+x_{2}^{2}+x_{3}^{2}+x_{4}^{2}=1\right\},
$$


we call it the lightcone unit sphere. We define the canonical lightcone projection $\pi: L C^{*} \longrightarrow S_{+}^{3}$ by

$$
\pi\left(x_{0}, x_{1}, x_{2}, x_{3}, x_{4}\right)=\left(1, \frac{x_{1}}{x_{0}}, \frac{x_{2}}{x_{0}}, \frac{x_{3}}{x_{0}}, \frac{x_{4}}{x_{0}}\right) .
$$

We also define the three-dimensional Euclidean unit 3-sphere in $\mathbb{R}_{0}^{4}$ by

$$
S_{0}^{3}=\left\{\boldsymbol{x} \in \mathbb{R}_{1}^{5} \mid x_{0}=0, x_{1}^{2}+x_{2}^{2}+x_{3}^{2}+x_{4}^{2}=1\right\},
$$

where $\mathbb{R}_{0}^{4}=\left\{\boldsymbol{x} \in \mathbb{R}_{1}^{5} \mid x_{0}=0\right\}$ is the Euclidean 4-space.

\section{CURVES IN THE UNIT 3-SPHERE AND FOCAL SURFACES}

Let $\boldsymbol{\gamma}: I \longrightarrow S_{+}^{3}$ be a regular curve. We have a map $\Phi: S_{+}^{3} \rightarrow S_{0}^{3}$ defined by $\Phi(\boldsymbol{v})=\boldsymbol{v}-\boldsymbol{e}_{0}$, which is an isometry. Then we have a regular curve $\bar{\gamma}: I \rightarrow S_{0}^{3}$ defined by $\bar{\gamma}(s)=\Phi(\gamma(s))$ $=\boldsymbol{\gamma}(s)-\boldsymbol{e}_{0}$, so that $\boldsymbol{\gamma}$ and $\bar{\gamma}$ have completely the same geometric properties as spherical curves. Since $\bar{\gamma}$ is a spacelike curve, we can reparameterize it by the arc-length $s$. So we have the unit tangent vector $\boldsymbol{t}(s)=\overline{\boldsymbol{\gamma}}^{\prime}(s)$ of $\overline{\boldsymbol{\gamma}}(s)$. Suppose that $\left\|\boldsymbol{t}^{\prime}(s)\right\| \neq 1$. Then $\left\|\boldsymbol{t}^{\prime}(s)+\overline{\boldsymbol{\gamma}}(s)\right\| \neq 0$, so that we have another unit vector $\boldsymbol{n}(s)=\frac{\boldsymbol{t}^{\prime}(s)+\bar{\gamma}(s)}{\left\|\boldsymbol{t}^{\prime}(s)+\bar{\gamma}(s)\right\|}$. We also define a unit vector by $\boldsymbol{b}(s)=\overline{\boldsymbol{\gamma}}(s) \wedge \boldsymbol{e}_{0} \wedge \boldsymbol{t}(s) \wedge \boldsymbol{n}(s)$, then we have a pseudo-orthonormal frame field $\{\overline{\boldsymbol{\gamma}}(s), \boldsymbol{t}(s), \boldsymbol{n}(s), \boldsymbol{b}(s)\}$ of $\mathbb{R}_{0}^{4}$ along $\overline{\boldsymbol{\gamma}}(s)$. By standard arguments, we have the following Frenet-Serret type formulae:

$$
\left\{\begin{array}{l}
\overline{\boldsymbol{\gamma}}^{\prime}(s)=\boldsymbol{t}(s) \\
\boldsymbol{t}^{\prime}(s)=\kappa_{g}(s) \boldsymbol{n}(s)-\overline{\boldsymbol{\gamma}}(s) \\
\boldsymbol{n}^{\prime}(s)=-\kappa_{g}(s) \boldsymbol{t}(s)+\tau_{g}(s) \boldsymbol{b}(s) \\
\boldsymbol{b}^{\prime}(s)=-\tau_{g}(s) \boldsymbol{n}(s)
\end{array}\right.
$$

where $\quad \kappa_{g}(s)=\left\|\boldsymbol{t}^{\prime}(s)+\overline{\boldsymbol{\gamma}}(s)\right\| \quad$ and $\quad \tau_{g}(s)=-\operatorname{det}\left(\overline{\boldsymbol{\gamma}}(s), \overline{\boldsymbol{\gamma}}^{\prime}(s), \overline{\boldsymbol{\gamma}}^{\prime \prime}(s), \overline{\boldsymbol{\gamma}}^{\prime \prime \prime}(s)\right) / \kappa_{g}^{2}(s)$. We call $\{\overline{\boldsymbol{\gamma}}, \boldsymbol{t}, \boldsymbol{n}, \boldsymbol{b}\}$ a Sabban frame of $\overline{\boldsymbol{\gamma}} \cdot{ }^{8}$ Here, $\kappa_{g}$ is called a geodesic curvature and $\tau_{g}$ a geodesic torsion of $\bar{\gamma}$ in $S_{0}^{3}$, respectively.

It is known that the evolute of the curve in the unit 2-sphere is the dual of the spherical curve given by the unit tangent vector $t(s) .{ }^{11}$ But for a curve in the unit 3 -sphere, the dual is a surface. We call it the focal surface of the original curve. We now consider the focal surface of a curve $\bar{\gamma}: I \rightarrow S_{0}^{3}$ analogous to the case for curves in Euclidean space. We define $F^{ \pm}: I \times J \rightarrow S_{0}^{3}$ by

$$
F^{ \pm}(s, u)=u \overline{\boldsymbol{\gamma}}(s)+\frac{u}{\kappa_{g}(s)} \boldsymbol{n}(s) \pm \frac{\sqrt{\kappa_{g}^{2}(s)-u^{2}\left(\kappa_{g}^{2}(s)+1\right)}}{\kappa_{g}(s)} \boldsymbol{b}(s) .
$$

We call each image of $F^{ \pm}$the spherical focal surface of $\bar{\gamma}$. We remark that the focal surfaces of $\bar{\gamma}$ satisfy the equations $\left\langle\bar{\gamma}^{\prime}(s), F^{ \pm}(s, u)\right\rangle=\left\langle\bar{\gamma}^{\prime \prime}(s), F^{ \pm}(s, u)\right\rangle=0$. This means that each one of the focal surfaces $F^{ \pm}(s, u)$ of $\boldsymbol{\gamma}$ is the spherical dual of $\boldsymbol{t}$ in the sense of Ref. 10. By straightforward calculations, we have

$$
\begin{aligned}
\frac{\partial F^{ \pm}}{\partial u}(s, u)= & \overline{\boldsymbol{\gamma}}(s)+\frac{1}{\kappa_{g}(s)} \boldsymbol{n}(s) \pm \frac{-u\left(\kappa_{g}^{2}(s)+1\right)}{\kappa_{g}(s) \sqrt{\kappa_{g}^{2}(s)-u^{2}\left(\kappa_{g}^{2}(s)+1\right)}} \boldsymbol{b}(s), \\
\frac{\partial F^{ \pm}}{\partial s}(s, u)= & -\frac{u \kappa_{g}^{\prime}(s) \pm \tau_{g}(s) \kappa_{g}(s) \sqrt{\kappa_{g}^{2}(s)-u^{2}\left(\kappa_{g}^{2}(s)+1\right)}}{\kappa_{g}^{2}(s)} \boldsymbol{n}(s) \\
& +\frac{u \tau_{g}(s) \kappa_{g}(s) \sqrt{\kappa_{g}^{2}(s)-u^{2}\left(\kappa_{g}^{2}(s)+1\right)} \pm u^{2} \kappa_{g}^{\prime}(s)}{\kappa_{g}^{2}(s) \sqrt{\kappa_{g}^{2}(s)-u^{2}\left(\kappa_{g}^{2}(s)+1\right)}} \boldsymbol{b}(s) .
\end{aligned}
$$


It follows that $\left\{\partial F^{ \pm} / \partial u, \partial F^{ \pm} / \partial s\right\}$ are linearly dependent if and only if

$$
\tau_{g}(s) \kappa_{g}(s) \sqrt{\kappa_{g}^{2}(s)-u^{2}\left(\kappa_{g}^{2}(s)+1\right)} \pm u \kappa_{g}^{\prime}(s)=0,
$$

so that we have

$$
u=\frac{\varepsilon_{i} \tau_{g}(s) \kappa_{g}^{2}(s)}{\sqrt{\kappa_{g}^{\prime 2}(s)+\kappa_{g}^{4}(s) \tau_{g}^{2}(s)+\kappa_{g}^{2}(s) \tau_{g}^{2}(s)}} \cdot\left(i=1,2 ; \varepsilon_{1}=1, \varepsilon_{2}=-1\right) .
$$

Therefore, each critical value set of $F^{ \pm}$is given by

$$
\boldsymbol{\varepsilon}_{\bar{\gamma}}^{ \pm}(s)=\frac{ \pm \tau_{g}(s) \kappa_{g}^{2}(s)}{\sqrt{\kappa_{g}^{\prime 2}(s)+\kappa_{g}^{4}(s) \tau_{g}^{2}(s)+\kappa_{g}^{2}(s) \tau_{g}^{2}(s)}}\left\{\overline{\boldsymbol{\gamma}}(s)+\frac{1}{\kappa_{g}(s)} \boldsymbol{n}(s)+\left(\frac{1}{\kappa_{g}(s)}\right)^{\prime} \frac{1}{\tau_{g}(s)} \boldsymbol{b}(s)\right\} .
$$

We remark that each curve of $\boldsymbol{\varepsilon}_{\bar{\gamma}}^{ \pm}$satisfies the equations

$$
\left\langle\overline{\boldsymbol{\gamma}}^{\prime}(s), \boldsymbol{\varepsilon}_{\bar{\gamma}}^{ \pm}(s)\right\rangle=\left\langle\overline{\boldsymbol{\gamma}}^{\prime \prime}(s), \boldsymbol{\varepsilon}_{\bar{\gamma}}^{ \pm}(s)\right\rangle=\left\langle\overline{\boldsymbol{\gamma}}^{\prime \prime \prime}(s), \boldsymbol{\varepsilon}_{\bar{\gamma}}^{ \pm}(s)\right\rangle=0 .
$$

In Ref. 11, Porteous introduced the notion of the evolute of $\bar{\gamma}$ in the unit 3 -sphere. He defined it as the curve which satisfies the above equations, so that we call each image of $\varepsilon_{\bar{\gamma}}^{ \pm}$the spherical evolute of $\bar{\gamma}$ in the unit 3-sphere. We remark that $\boldsymbol{\varepsilon}_{\bar{\gamma}}^{-}(s)=-\boldsymbol{\varepsilon}_{\bar{\gamma}}^{+}(s)$. For $s=s_{0}$, we fix $\boldsymbol{v}_{0}^{ \pm}=\boldsymbol{\varepsilon}_{\bar{\gamma}}^{ \pm}\left(s_{0}\right)$ and $\left\langle\bar{\gamma}\left(s_{0}\right), \boldsymbol{\varepsilon}_{\bar{\gamma}}^{ \pm}\left(s_{0}\right)\right\rangle=c^{ \pm}$. Since $\boldsymbol{v}_{0}^{-}=-\boldsymbol{v}_{0}^{+}$and $c^{-}=-c^{+}$, we have a hyperplane

$$
H P\left(\boldsymbol{v}_{0}^{+}, c^{+}\right)=\left\{\boldsymbol{x} \in \mathbb{R}_{0}^{4} \mid\left\langle\boldsymbol{x}, \boldsymbol{v}_{0}^{+}\right\rangle=c^{+}\right\}=\left\{\boldsymbol{x} \in \mathbb{R}_{0}^{4} \mid\left\langle\boldsymbol{x}, \boldsymbol{v}_{0}^{-}\right\rangle=c^{-}\right\}=H P\left(\boldsymbol{v}_{0}^{-}, c^{-}\right),
$$

so that we have a sphere

$$
S^{2}\left(\boldsymbol{v}_{0}^{ \pm}, c^{ \pm}\right)=H P\left(\boldsymbol{v}_{0}^{ \pm}, c^{ \pm}\right) \cap S_{0}^{3} .
$$

We call $S^{2}\left(\boldsymbol{v}_{0}^{ \pm}, c^{ \pm}\right)$an osculating sphere of $\bar{\gamma}$ at $s_{0}$. Therefore, the spherical evolutes $\boldsymbol{\varepsilon}_{\bar{\gamma}}^{ \pm}(s)$ are the loci of the centers of osculating spheres of $\bar{\gamma}$.

Proposition 3.1. There exists a sphere $S^{2}(\boldsymbol{v}, c) \subset S_{0}^{3}$ such that $\overline{\boldsymbol{\gamma}}(I) \subset S^{2}(\boldsymbol{v}, c)$ if and only if both of the spherical evolutes $\boldsymbol{\varepsilon}_{\bar{\gamma}}^{ \pm}$of $\overline{\boldsymbol{\gamma}}$ are single points.

Proof. If one of the spherical evolutes $\boldsymbol{\varepsilon}_{\bar{\gamma}}^{+}$of $\overline{\boldsymbol{\gamma}}$ is a single point, we can set that $\boldsymbol{\varepsilon}_{\bar{\gamma}}^{+}(s)=\boldsymbol{v}^{+}$. In this case another spherical evolute $\boldsymbol{\varepsilon}_{\bar{\gamma}}^{-}$is constant too. Then $\left\langle\overline{\boldsymbol{\gamma}}(s), \boldsymbol{v}^{+}\right\rangle^{\prime}=\left\langle\overline{\boldsymbol{\gamma}}^{\prime}(s), \boldsymbol{v}^{+}\right\rangle=\left\langle\boldsymbol{t}(s), \boldsymbol{\varepsilon}_{\bar{\gamma}}^{+}(s)\right\rangle$ $=0$, so we have $\left\langle\overline{\boldsymbol{\gamma}}(s), \boldsymbol{v}^{+}\right\rangle=c^{+}$and $\overline{\boldsymbol{\gamma}}(I) \subset S^{2}\left(\boldsymbol{v}^{+}, c^{+}\right)$. Conversely, if $\overline{\boldsymbol{\gamma}}(I) \subset S^{2}(\boldsymbol{v}, c)$, then at any point on $\bar{\gamma}$, the osculating spheres is $S^{2}(\boldsymbol{v}, c)$ itself. So the locus of the centers of osculating spheres of $\bar{\gamma}$ is $\boldsymbol{v}$ and $\boldsymbol{- v}$. Therefore, both of the spherical evolutes $\boldsymbol{\varepsilon}_{\bar{\gamma}}^{ \pm}$of $\bar{\gamma}$ are single points. This completes the proof.

\section{LIGHTCONE DUALS OF CURVES IN THE UNIT 3-SPHERE}

In Ref. 5, the first author introduced the Legendrian dualities between pseudo-spheres in Lorentz-Minkowski 5-space which is a basic tool for the study of hypersurfaces in pseudo-spheres in Lorentz-Minkowski 5-space. We define one-forms $\langle d \boldsymbol{v}, \boldsymbol{w}\rangle=-w_{0} d v_{0}+\sum_{i=1}^{4} w_{i} d v_{i},\langle\boldsymbol{v}, d \boldsymbol{w}\rangle$ $=-v_{0} d w_{0}+\sum_{i=1}^{4} v_{i} d w_{i}$ in $\mathbb{R}_{1}^{5} \times \mathbb{R}_{1}^{5}$ and consider the following two double fibrations:

(1) (a) $L C^{*} \times S_{1}^{4} \supset \Delta_{3}=\{(\boldsymbol{v}, \boldsymbol{w}) \mid\langle\boldsymbol{v}, \boldsymbol{w}\rangle=1\}$,

(b) $\pi_{31}: \Delta_{3} \longrightarrow L C^{*}, \pi_{32}: \Delta_{3} \longrightarrow S_{1}^{4}$,

(c) $\theta_{31}=\langle d \boldsymbol{v}, \boldsymbol{w}\rangle\left|\Delta_{3}, \theta_{32}=\langle\boldsymbol{v}, d \boldsymbol{w}\rangle\right| \Delta_{3}$.

(2) (a) $L C^{*} \times L C^{*} \supset \Delta_{4}=\{(\boldsymbol{v}, \boldsymbol{w}) \mid\langle\boldsymbol{v}, \boldsymbol{w}\rangle=-2\}$,

(b) $\pi_{41}: \Delta_{4} \longrightarrow L C^{*}, \pi_{42}: \Delta_{4} \longrightarrow L C^{*}$,

(c) $\theta_{41}=\langle d \boldsymbol{v}, \boldsymbol{w}\rangle\left|\Delta_{4}, \theta_{42}=\langle\boldsymbol{v}, d \boldsymbol{w}\rangle\right| \Delta_{4}$.

Here, $\pi_{i 1}(\boldsymbol{v}, \boldsymbol{w})=\boldsymbol{v}, \pi_{i 2}(\boldsymbol{v}, \boldsymbol{w})=\boldsymbol{w}$. We remark that $\theta_{i 1}^{-1}(0)$ and $\theta_{i 2}^{-1}(0)$ define the same tangent hyperplane field over $\Delta_{i}$ which is denoted by $K_{i}, \mathrm{i}=3,4$. It has been shown in Ref. 5 that each 
$\left(\Delta_{i}, K_{i}\right)(i=3,4)$ is a contact manifold and both of $\pi_{i j}(j=1,2)$ are Legendrian fibrations. Moreover, those contact manifolds are contact diffeomorphic to each other. In Ref. 5, we have defined four double fibrations $\left(\Delta_{i}, K_{i}\right), i=1,2,3,4$, such that these are contact diffeomorphic to each other. Here, we only use $\left(\Delta_{3}, K_{3}\right)$ and $\left(\Delta_{4}, K_{4}\right)$. If we have an isotropic mapping $i: L \rightarrow \Delta_{i}$ (i.e., $i^{*} \theta_{i 1}$ $=0$ ), we say that $\pi_{i 1}(i(L))$ and $\pi_{i 2}(i(L))$ are $\Delta_{i}$-dual to each other, $i=3,4$. For detailed properties of Legendrian fibrations, see Ref. 1.

We now define hypersurfaces in $L C^{*}$ associated with the curves in $S_{+}^{3}$ or $S_{0}^{3}$. Let $\boldsymbol{\gamma}: I \longrightarrow S_{+}^{3}$ be a unit speed curve. We define $\overline{L D}_{\bar{\gamma}}^{ \pm}: I \times \mathbb{R}^{2} \longrightarrow L C^{*}$ by

$$
\overline{L D}_{\bar{\gamma}}^{ \pm}(s, u, v)=\overline{\boldsymbol{\gamma}}(s)+u \boldsymbol{n}(s)+v \boldsymbol{b}(s) \pm \sqrt{u^{2}+v^{2}+1} \boldsymbol{e}_{0}
$$

We also define $L D_{\gamma}: I \times \mathbb{R}^{2} \longrightarrow L C^{*}$ by

$$
L D_{\gamma}(s, u, v)=\frac{u^{2}+v^{2}-4}{4} \overline{\boldsymbol{\gamma}}(s)+u \boldsymbol{n}(s)+v \boldsymbol{b}(s)+\frac{u^{2}+v^{2}+4}{4} \boldsymbol{e}_{0} .
$$

Then we have the following proposition.

Proposition 4.1. Under the above notation, we have the followings:

(1) $\bar{\gamma}$ and $\overline{L D_{\bar{\gamma}}^{ \pm}}$are $\Delta_{3}$-dual to each other.

(2) $\gamma$ and $L D_{\gamma}$ are $\Delta_{4}$-dual to each other.

Proof. Consider the mapping $\mathscr{L}_{3}(s, u, v)=\left(\overline{L D}_{\bar{\gamma}}^{ \pm}(s, u, v), \bar{\gamma}(s)\right)$. Then we have

$$
\left\langle\overline{L D}_{\bar{\gamma}}^{ \pm}(s, u, v), \bar{\gamma}(s)\right\rangle=\langle\bar{\gamma}(s), \bar{\gamma}(s)\rangle=1
$$

and

$$
\mathscr{L}_{3}^{*} \theta_{32}=\left\langle\overline{L D}_{\bar{\gamma}}^{ \pm}(s, u, v), \overline{\boldsymbol{\gamma}}^{\prime}(s)\right\rangle d s=\left\langle\overline{L D}_{\bar{\gamma}}^{ \pm}(s, u, v), \boldsymbol{t}(s)\right\rangle d s=0 .
$$

The assertion (1) holds.

We also consider the mapping $\mathscr{L}_{4}(s, u, v)=\left(L D_{\gamma}(s, u, v), \boldsymbol{\gamma}(s)\right)$. Since $\left\langle\boldsymbol{\gamma}(s), \boldsymbol{e}_{0}\right\rangle=-1$ and $\langle\boldsymbol{\gamma}(s), \overline{\boldsymbol{\gamma}}(s)\rangle=1$, we have $\left\langle L D_{\boldsymbol{\gamma}}(s, u, v), \boldsymbol{\gamma}(s)\right\rangle=\left(u^{2}+v^{2}\right) / 4-1-\left(\left(u^{2}+v^{2}\right) / 4+1\right)=-2$. Moreover, we have

$$
\left.\mathscr{L}_{4}^{*} \theta_{42}=\left\langle L D_{\gamma}(s, u, v)\right), \boldsymbol{\gamma}^{\prime}(s)\right\rangle d s=\left\langle L D_{\gamma}(s, u, v), \boldsymbol{t}(s)\right\rangle d s=0 .
$$

This completes the proof.

We call each image of $\overline{L D}_{\bar{\gamma}}^{ \pm}$the Lightcone dual haypersurface of the de Sitter spherical curve $\bar{\gamma}$ and $L D_{\gamma}$ the Lightcone dual hypersurface of the lightlike spherical curve $\gamma$. Then we have two mappings $\pi \circ \overline{L D}_{\bar{\gamma}}^{ \pm}: I \times \mathbb{R}^{2} \rightarrow S_{+}^{3}$ and $\pi \circ L D_{\gamma}: I \times \mathbb{R}^{2} \rightarrow S_{+}^{3}$ defined by

$$
\begin{aligned}
& \pi \circ \overline{L D}_{\bar{\gamma}}^{ \pm}(s, u, v)= \pm\left(\frac{1}{\sqrt{u^{2}+v^{2}+1}} \overline{\boldsymbol{\gamma}}(s)+\frac{u}{\sqrt{u^{2}+v^{2}+1}} \boldsymbol{n}(s)+\frac{v}{\sqrt{u^{2}+v^{2}+1}} \boldsymbol{b}(s)\right)+\boldsymbol{e}_{0}, \\
& \pi \circ L D_{\boldsymbol{\gamma}}(s, u, v)=\frac{u^{2}+v^{2}-4}{u^{2}+v^{2}+4} \bar{\gamma}(s)+\frac{4 u}{u^{2}+v^{2}+4} \boldsymbol{n}(s)+\frac{4 v}{u^{2}+v^{2}+4} \boldsymbol{b}(s)+\boldsymbol{e}_{0} .
\end{aligned}
$$

In this paper, we consider the singularities of these dual surfaces and mappings. By the FrenetSerret type formulae, we have

$$
\begin{gathered}
\frac{\partial \overline{L D}_{\bar{\gamma}}^{ \pm}}{\partial u}(s, u, v)=\boldsymbol{n}(s) \pm \frac{u}{\sqrt{1+u^{2}+v^{2}}} \boldsymbol{e}_{0}, \\
\frac{\partial \overline{L D_{\bar{\gamma}}^{ \pm}}}{\partial v}(s, u, v)=\boldsymbol{b}(s) \pm \frac{v}{\sqrt{1+u^{2}+v^{2}}} \boldsymbol{e}_{0},
\end{gathered}
$$




$$
\begin{aligned}
& \frac{\partial \overline{L D}_{\bar{\gamma}}^{ \pm}}{\partial s}(s, u, v)=\left(1-u \kappa_{g}(s)\right) \boldsymbol{t}(s)-v \tau_{g}(s) \boldsymbol{n}(s)+u \tau_{g}(s) \boldsymbol{b}(s), \\
& \frac{\partial L D_{\gamma}}{\partial u}(s, u, v)=\frac{u}{2} \bar{\gamma}(s)+\boldsymbol{n}(s)+\frac{u}{2} \boldsymbol{e}_{0}, \\
& \frac{\partial L D_{\gamma}}{\partial v}(s, u, v)=\frac{v}{2} \bar{\gamma}(s)+\boldsymbol{b}(s)+\frac{v}{2} \boldsymbol{e}_{0}, \\
& \frac{\partial L D_{\gamma}}{\partial s}(s, u, v)=\frac{u^{2}+v^{2}-4 u \kappa_{g}(s)-4}{4} \boldsymbol{t}(s)-v \tau_{g}(s) \boldsymbol{n}(s)+u \tau_{g}(s) \boldsymbol{b}(s) .
\end{aligned}
$$

Then we have the following proposition.

Proposition 4.2. Let $\boldsymbol{\gamma}: I \longrightarrow S_{+}^{3}$ be a unit speed curve. Then we have the followings:

(1) $(s, u, v)$ is a singular point of $\bar{L}_{\bar{\gamma}}^{ \pm}$if and only if $u=1 / \kappa_{g}(s)$.

(2) $(s, u, v)$ is a singular point of $L D_{\gamma}$ if and only if $v= \pm \sqrt{4+4 u \kappa_{g}(s)-u^{2}}$.

Proof. By the above calculations, $\partial \bar{L}_{\bar{\gamma}}^{ \pm} / \partial u(s, u, v), \partial \bar{L}_{\bar{\gamma}}^{ \pm} / \partial v(s, u, v), \partial \bar{L}_{\bar{\gamma}}^{ \pm} / \partial s(s, u, v)$ are linearly dependent if and only if $u=1 / \kappa_{g}(s)$. The assertion (1) follows. By the similar reason, we have the assertion (2). This completes the proof.

Therefore, the critical value sets of the above dual hypersurfaces are given by

$$
\begin{aligned}
C\left(\bar{L} \bar{D}_{\bar{\gamma}}^{ \pm}\right) & =\left\{\bar{\gamma}(s)+\frac{1}{\kappa_{g}(s)} \boldsymbol{n}(s)+v \boldsymbol{b}(s) \pm \sqrt{\frac{1+\kappa_{g}^{2}(s)+v^{2} \kappa_{g}^{2}(s)}{\kappa_{g}^{2}(s)}} \boldsymbol{e}_{0} \mid v \in \mathbb{R}, s \in I, \kappa_{g}(s) \neq 0\right\}, \\
C\left(L D_{\gamma}\right)^{ \pm} & =\left\{\kappa_{g}(s) u \bar{\gamma}(s)+u \boldsymbol{n}(s) \pm \sqrt{4+4 u \kappa_{g}(s)-u^{2}} \boldsymbol{b}(s)+\left(\kappa_{g}(s) u+2\right) \boldsymbol{e}_{0} \mid u \in \mathbb{R}, s \in I\right\} .
\end{aligned}
$$

We, respectively, denote that

$$
\begin{aligned}
& L F_{\bar{\gamma}}^{ \pm}(s, u)=\bar{\gamma}(s)+\frac{1}{\kappa_{g}(s)} \boldsymbol{n}(s)+v(u) \boldsymbol{b}(s) \pm \sqrt{\frac{1+\kappa_{g}^{2}(s)+v^{2}(u) \kappa_{g}^{2}(s)}{\kappa_{g}^{2}(s)}} \boldsymbol{e}_{0}, \\
& L F_{\gamma}^{ \pm}(s, u)=\kappa_{g}(s) u \bar{\gamma}(s)+u \boldsymbol{n}(s) \pm \sqrt{4+4 u \kappa_{g}(s)-u^{2}} \boldsymbol{b}(s)+\left(\kappa_{g}(s) u+2\right) \boldsymbol{e}_{0}
\end{aligned}
$$

where we have the relation $v(u)= \pm \sqrt{4+4 u \kappa_{g}(s)-u^{2}}$. We respectively call each one of $L F_{\bar{\gamma}}^{ \pm}$the lightcone focal surface of the de Sitter spherical curve $\bar{\gamma}$ and each one of $L F_{\gamma}^{ \pm}$the ligtcone focal surface of the lightcone spherical curve $\gamma$. Then the projections of $L F_{\bar{\gamma}}^{ \pm}$and $L F_{\gamma}^{ \pm}$to $S_{+}^{3}$ are given as follows:

$$
\begin{aligned}
& \pi\left(C\left(\overline{L D_{\bar{\gamma}}^{ \pm}}\right)\right)=\left\{\frac{ \pm\left(\kappa_{g}(s) \overline{\boldsymbol{\gamma}}(s)+\boldsymbol{n}(s)+v \kappa_{g}(s) \boldsymbol{b}(s)\right)}{\sqrt{1+\kappa_{g}^{2}(s)+v^{2} \kappa_{g}^{2}(s)}}+\boldsymbol{e}_{0} \mid v \in \mathbb{R}, s \in I, \kappa_{g}(s) \neq 0\right\}, \\
& \pi\left(C\left(L D_{\boldsymbol{\gamma}}\right)^{ \pm}\right)=\left\{\frac{u \kappa_{g}(s) \overline{\boldsymbol{\gamma}}(s)+u \boldsymbol{n}(s) \pm \sqrt{4+4 u \kappa_{g}(s)-u^{2}} \boldsymbol{b}(s)}{\kappa_{g}(s) u+2}+\boldsymbol{e}_{0} \mid u \in \mathbb{R}, s \in I\right\} .
\end{aligned}
$$

On the other hand, we define $\tilde{\pi}=\Phi \circ \pi: L C^{*} \rightarrow S_{0}^{3}$. By the previous calculations, $\tilde{\pi}\left(C\left(\overline{L D}_{\bar{\gamma}}^{ \pm}\right)\right)$ is different from $\tilde{\pi}\left(C\left(L D_{\gamma}\right)^{ \pm}\right)$. In Ref. 7, it was shown that the projections of the critical value sets of the lightcone dual hypersurfaces of $\gamma$ and $\bar{\gamma}$ are the same for a curve $\gamma: I \rightarrow S_{+}^{2}$. Moreover, it is equal to the spherical evolute of $\bar{\gamma}$. Therefore, the situation for curves in $S_{+}^{3}$ is quite different from that for curves in $S_{+}^{2}$. 


\section{LIGHTCONE HEIGHT FUNCTIONS}

In order to study the singularities of Lightcone dual surfaces of spherical curves, we introduce two families of functions and apply the theory of unfoldings. Let $\gamma: I \longrightarrow S_{+}^{3}$ be a unit speed curve, according to the duality between lightcone and de Sitter 4-space in Sec. IV, we define the following family of functions:

$$
\bar{H}: I \times L C^{*} \longrightarrow \mathbb{R}, \bar{H}(s, \boldsymbol{v})=\langle\bar{\gamma}(s), \boldsymbol{v}\rangle-1 .
$$

We call $\bar{H}$ a lightcone height function of the de Sitter spherical curve $\bar{\gamma}$. For any fixed $v \in L C^{*}$, we denote $\bar{h}_{v}(s)=\bar{H}(s, v)$. For simplification, we denote

$$
\rho(s)=\sqrt{\left(\kappa_{g}^{4}(s) \tau_{g}^{2}(s)+\kappa_{g}^{2}(s) \tau_{g}^{2}(s)+\kappa_{g}^{\prime 2}(s)\right) / \kappa_{g}^{4}(s) \tau_{g}^{2}(s)} .
$$

Then we have the following proposition for $\bar{h}_{v}$.

Proposition 5.1. Let $\boldsymbol{\gamma}: I \longrightarrow S_{+}^{3}$ be a unit speed curve, then we have the followings:

(1) $\bar{h}_{v}(s)=0$ if and only if there exist $\lambda, \mu, \xi, \eta \in \mathbb{R}$ with $\eta^{2}=1+\lambda^{2}+\mu^{2}+\xi^{2}$ such that $\boldsymbol{v}=\overline{\boldsymbol{\gamma}}(s)+\lambda \boldsymbol{t}(s)+\mu \boldsymbol{n}(s)+\xi \boldsymbol{b}(s)+\eta \boldsymbol{e}_{0}$.

(2) $\bar{h}_{v}(s)=\bar{h}_{v}^{\prime}(s)=0$ if and only if there exist $\mu, \xi, \eta \in \mathbb{R}$ with $\eta^{2}=1+\mu^{2}+\xi^{2}$ such that $\boldsymbol{v}=\overline{\boldsymbol{\gamma}}(s)+\mu \boldsymbol{n}(s)+\xi \boldsymbol{b}(s)+\eta \boldsymbol{e}_{0}=\overline{\boldsymbol{\gamma}}(s)+\mu \boldsymbol{n}(s)+\xi \boldsymbol{b}(s) \pm \sqrt{1+\mu^{2}+\xi^{2}} \boldsymbol{e}_{0}$.

(3) $\bar{h}_{\boldsymbol{v}}(s)=\bar{h}_{\boldsymbol{v}}^{\prime}(s)=\bar{h}_{\boldsymbol{v}}^{\prime \prime}(s)=0$ if and only if $\kappa_{g}(s) \neq 0$ and

$$
\boldsymbol{v}=\overline{\boldsymbol{\gamma}}(s)+\frac{1}{\kappa_{g}(s)} \boldsymbol{n}(s)+\xi \boldsymbol{b}(s) \pm \sqrt{\frac{1+\kappa_{g}^{2}(s)+\kappa_{g}^{2}(s) \xi^{2}}{\kappa_{g}^{2}(s)}} \boldsymbol{e}_{0} .
$$

(4) $\bar{h}_{\boldsymbol{v}}(s)=\bar{h}_{\boldsymbol{v}}^{\prime}(s)=\bar{h}_{\boldsymbol{v}}^{\prime \prime}(s)=\bar{h}_{\boldsymbol{v}}^{\prime \prime \prime}(s)=0$ if and only if $\kappa_{g}(s) \neq 0, \tau_{g}(s) \neq 0$ and

$$
\boldsymbol{v}=\overline{\boldsymbol{\gamma}}(s)+\frac{1}{\kappa_{g}(s)} \boldsymbol{n}(s)-\frac{\kappa_{g}^{\prime}(s)}{\kappa_{g}^{2}(s) \tau_{g}(s)} \boldsymbol{b}(s) \pm \rho(s) \boldsymbol{e}_{0} .
$$

(5) $\bar{h}_{\boldsymbol{v}}(s)=\bar{h}_{\boldsymbol{v}}^{\prime}(s)=\bar{h}_{\boldsymbol{v}}^{\prime \prime}(s)=\bar{h}_{\boldsymbol{v}}^{\prime \prime \prime}(s)=\bar{h}_{\boldsymbol{v}}^{(4)}(s)=0$ if and only if $\kappa_{g}(s) \neq 0, \tau_{g}(s) \neq 0$,

$$
\left(\left(\frac{-1}{\kappa_{g}(s)}\right)^{\prime} \frac{1}{\tau_{g}(s)}\right)^{\prime}-\frac{\tau_{g}(s)}{\kappa_{g}(s)}=0
$$

and

$$
\boldsymbol{v}=\overline{\boldsymbol{\gamma}}(s)+\frac{1}{\kappa_{g}(s)} \boldsymbol{n}(s)-\frac{\kappa_{g}^{\prime}(s)}{\kappa_{g}^{2}(s) \tau_{g}(s)} \boldsymbol{b}(s) \pm \rho(s) \boldsymbol{e}_{0} .
$$

(6) $\bar{h}_{\boldsymbol{v}}(s)=\bar{h}_{\boldsymbol{v}}^{\prime}(s)=\bar{h}_{\boldsymbol{v}}^{\prime \prime}(s)=\bar{h}_{\boldsymbol{v}}^{\prime \prime \prime}(s)=\bar{h}_{\boldsymbol{v}}^{(4)}(s)=\bar{h}_{\boldsymbol{v}}^{(5)}(s)=0$ if and only if $\kappa_{g}(s) \neq 0, \tau_{g}(s) \neq 0$,

$$
\left(\left(\frac{-1}{\kappa_{g}(s)}\right)^{\prime} \frac{1}{\tau_{g}(s)}\right)^{\prime}-\frac{\tau_{g}(s)}{\kappa_{g}(s)}=\left\{\left(\left(\frac{-1}{\kappa_{g}(s)}\right)^{\prime} \frac{1}{\tau_{g}(s)}\right)^{\prime}-\frac{\tau_{g}(s)}{\kappa_{g}(s)}\right\}^{\prime}=0
$$

and

$$
\boldsymbol{v}=\overline{\boldsymbol{\gamma}}(s)+\frac{1}{\kappa_{g}(s)} \boldsymbol{n}(s)-\frac{\kappa_{g}^{\prime}(s)}{\kappa_{g}^{2}(s) \tau_{g}(s)} \boldsymbol{b}(s) \pm \rho(s) \boldsymbol{e}_{0} .
$$

Proof. (1) Since $v \in L C^{*}$, there exist $\omega, \lambda, \mu, \xi, \eta \in \mathbb{R}$ with $\omega^{2}+\lambda^{2}+\mu^{2}+\xi^{2}-\eta^{2}$ $=0$ such that $\boldsymbol{v}=\omega \overline{\boldsymbol{\gamma}}(s)+\lambda \boldsymbol{t}(s)+\mu \boldsymbol{n}(s)+\xi \boldsymbol{b}(s)+\eta \boldsymbol{e}_{0}$. From $\bar{h}_{\boldsymbol{v}}(s)=\langle\overline{\boldsymbol{\gamma}}(s), \boldsymbol{v}\rangle-1=0$, we have $\omega=1$. So $\boldsymbol{v}=\overline{\boldsymbol{\gamma}}(s)+\lambda \boldsymbol{t}(s)+\mu \boldsymbol{n}(s)+\xi \boldsymbol{b}(s)+\eta \boldsymbol{e}_{0}$ and $\eta^{2}=1+\lambda^{2}+\mu^{2}+\xi^{2}$. The converse direction also holds.

(2) Since $\bar{h}_{\boldsymbol{v}}^{\prime}(s)=\langle\boldsymbol{t}(s), \boldsymbol{v}\rangle, \bar{h}_{\boldsymbol{v}}(s)=\bar{h}_{\boldsymbol{v}}^{\prime}(s)=0$ if and only if

$$
\bar{h}_{\boldsymbol{v}}^{\prime}(s)=\langle\boldsymbol{t}(s), \boldsymbol{v}\rangle=\left\langle\boldsymbol{t}(s), \overline{\boldsymbol{\gamma}}(s)+\lambda \boldsymbol{t}(s)+\mu \boldsymbol{n}(s)+\xi \boldsymbol{b}(s)+\eta \boldsymbol{e}_{0}\right\rangle=\lambda=0 .
$$


It follows from the fact $\eta^{2}=1+\mu^{2}+\xi^{2}$ that $\eta= \pm \sqrt{1+\mu^{2}+\xi^{2}}$. Then we have $v=\bar{\gamma}(s)$ $+\mu \boldsymbol{n}(s)+\xi \boldsymbol{b}(s)+\eta \boldsymbol{e}_{0}=\overline{\boldsymbol{\gamma}}(s)+\mu \boldsymbol{n}(s)+\xi \boldsymbol{b}(s) \pm \sqrt{1+\mu^{2}+\xi^{2}} \boldsymbol{e}_{0}$.

(3) Since $\bar{h}_{\boldsymbol{v}}^{\prime \prime}(s)=\left\langle\kappa_{g}(s) \boldsymbol{n}(s)-\overline{\boldsymbol{\gamma}}(s), \boldsymbol{v}\right\rangle, \bar{h}_{\boldsymbol{v}}(s)=\bar{h}_{\boldsymbol{v}}^{\prime}(s)=\bar{h}_{\boldsymbol{v}}^{\prime \prime}(s)=0$ if and only if

$$
\bar{h}_{\boldsymbol{v}}^{\prime \prime}(s)=\left\langle\kappa_{g}(s) \boldsymbol{n}(s)-\overline{\boldsymbol{\gamma}}(s), \overline{\boldsymbol{\gamma}}(s)+\mu \boldsymbol{n}(s)+\xi \boldsymbol{b}(s) \pm \sqrt{1+\mu^{2}+\xi^{2}} \boldsymbol{e}_{0}\right\rangle=\kappa_{g}(s) \mu-1=0 .
$$

Then we have $\kappa_{g}(s) \neq 0, \mu=1 / \kappa_{g}(s)$ and

$$
\boldsymbol{v}=\overline{\boldsymbol{\gamma}}(s)+\boldsymbol{n}(s) / \kappa_{g}(s)+\xi \boldsymbol{\xi}(s) \pm \sqrt{\left(1+\kappa_{g}^{2}(s)+\kappa_{g}^{2}(s) \xi^{2}\right) / \kappa_{g}^{2}(s)} \boldsymbol{e}_{0} .
$$

(4) Since $\bar{h}_{\boldsymbol{v}}^{\prime \prime \prime}(s)=\left\langle\kappa_{g}^{\prime}(s) \boldsymbol{n}(s)-\left(\kappa_{g}^{2}(s)+1\right) \boldsymbol{t}(s)+\kappa_{g}(s) \tau_{g}(s) \boldsymbol{b}(s), \boldsymbol{v}\right\rangle, \bar{h}_{\boldsymbol{v}}(s)=\bar{h}_{\boldsymbol{v}}^{\prime}(s)=\bar{h}_{\boldsymbol{v}}^{\prime \prime}(s)$ $=\bar{h}_{v}^{\prime \prime \prime}(s)=0$ if and only if

$$
\begin{aligned}
\bar{h}_{\boldsymbol{v}}^{\prime \prime \prime}(s)= & \left\langle\kappa_{g}^{\prime}(s) \boldsymbol{n}(s)-\left(\kappa_{g}^{2}(s)+1\right) \boldsymbol{t}(s)+\kappa_{g}(s) \tau_{g}(s) \boldsymbol{b}(s),\right. \\
& \left.\overline{\boldsymbol{\gamma}}(s)+\boldsymbol{n}(s) / \kappa_{g}(s)+\xi \boldsymbol{b}(s) \pm \sqrt{\left(1+\kappa_{g}^{2}(s)+\kappa_{g}^{2}(s) \xi^{2}\right) / \kappa_{g}^{2}(s)} \boldsymbol{e}_{0}\right\rangle \\
= & \kappa_{g}^{\prime}(s) / \kappa_{g}(s)+\kappa_{g}(s) \tau_{g}(s) \xi=0 .
\end{aligned}
$$

Then we have $\kappa_{g}(s) \neq 0, \tau_{g}(s) \neq 0, \xi=-\kappa_{g}^{\prime}(s) / \kappa_{g}^{2}(s) \tau_{g}(s)$, and $\boldsymbol{v}=\overline{\boldsymbol{\gamma}}(s)+\boldsymbol{n}(s) / \kappa_{g}(s)$ $-\kappa_{g}^{\prime}(s) \boldsymbol{b}(s) / \kappa_{g}^{2}(s) \tau_{g}(s) \pm \rho(s) \boldsymbol{e}_{0}$.

The proof of (5) and (6) follows by computing the 4th and 5th derivatives of $\bar{h}_{v}(s)$ with respect to $s$. The calculations are lengthy and are omitted.

For a function $f(s)$, we say that $f$ has $A_{k}$-singularity at $s_{0}$ if $f^{(p)}\left(s_{0}\right)=0$ for all $1 \leq p \leq k$ and $f^{(k+1)}\left(s_{0}\right) \neq 0$. By Proposition 5.1 (6), we know that for $\bar{h}_{v}$, only $A_{k}$-singularity, $k=1,2,3,4$, are generic singularities.

Let $\gamma: I \longrightarrow S_{+}^{3}$ be a unit speed curve, according to the duality in the lightcone in Sec. IV, we define the following family of functions:

$$
H: I \times L C^{*} \longrightarrow \mathbb{R}, H(s, \boldsymbol{v})=\langle\boldsymbol{\gamma}(s), \boldsymbol{v}\rangle+2 .
$$

We call $H$ a lightcone height function of the lightlike spherical curve $\gamma$. For any fixed $v \in L C^{*}$, we denote $h_{v}(s)=H(s, \boldsymbol{v})$. For simplification, we denote

$$
\sigma^{ \pm}(s)=\left(\kappa_{g}^{2}(s) \tau_{g}(s) \pm \sqrt{\kappa_{g}^{\prime 2}(s)+\kappa_{g}^{2}(s) \tau_{g}^{2}(s)+\kappa_{g}^{4}(s) \tau_{g}^{2}(s)}\right) /\left(\kappa_{g}^{\prime 2}(s)+\kappa_{g}^{2}(s) \tau_{g}^{2}(s)\right) .
$$

The we have the following proposition for $h_{v}$.

Proposition 5.2. Let $\gamma: I \longrightarrow S_{+}^{3}$ be a unit speed curve, then we have the followings:

(1) $h_{\boldsymbol{v}}(s)=0$ if and only if $\boldsymbol{v}=\lambda \overline{\boldsymbol{\gamma}}(s)+\mu \boldsymbol{t}(s)+\xi \boldsymbol{n}(s)+\eta \boldsymbol{b}(s)+(\lambda+2) \boldsymbol{e}_{0}$, where $\lambda, \mu, \xi, \eta$ $\in \mathbb{R}$ and $\mu^{2}+\xi^{2}+\eta^{2}-4 \lambda-4=0$.

(2) $h_{v}(s)=h_{\boldsymbol{v}}^{\prime}(s)=0$ if and only if $\boldsymbol{v}=\left(\left(\xi^{2}+\eta^{2}\right) / 4-1\right) \overline{\boldsymbol{\gamma}}(s)+\xi \boldsymbol{n}+\eta \boldsymbol{b}(s)+\left(\left(\xi^{2}+\eta^{2}\right) / 4\right.$ $+1) \boldsymbol{e}_{0}$.

(3) $h_{v}(s)=h_{v}^{\prime}(s)=h_{v}^{\prime \prime}(s)=0$ if and only if

$$
\boldsymbol{v}=\kappa_{g}(s) \xi \overline{\boldsymbol{\gamma}}(s)+\xi \boldsymbol{n}(s) \pm \sqrt{4+4 \kappa_{g}(s) \xi-\xi^{2}} \boldsymbol{b}(s)+\left(\kappa_{g}(s) \xi+2\right) \boldsymbol{e}_{0} .
$$

(4) $h_{v}(s)=h_{v}^{\prime}(s)=h_{v}^{\prime \prime}(s)=h_{v}^{\prime \prime \prime}(s)=0$ if and only if $\kappa_{g}^{\prime 2}(s)+\kappa_{g}^{2}(s) \tau_{g}^{2}(s) \neq 0$ and $\boldsymbol{v}=2 \kappa_{g}^{2}(s) \tau_{g}(s) \sigma^{ \pm}(s) \bar{\gamma}(s)+2 \kappa_{g}(s) \tau_{g}(s) \sigma^{ \pm}(s) \boldsymbol{n}(s)-2 \kappa_{g}^{\prime}(s) \sigma^{ \pm}(s) \boldsymbol{b}(s)+\left(2 \kappa_{g}^{2}(s) \tau_{g}(s) \sigma^{ \pm}(s)+2\right) \boldsymbol{e}_{0}$.

(5) $h_{v}(s)=h_{v}^{\prime}(s)=h_{v}^{\prime \prime}(s)=h_{v}^{\prime \prime \prime}(s)=h_{v}^{(4)}(s)=0$ if and only if $\kappa_{g}^{\prime 2}(s)+\kappa_{g}^{2}(s) \tau_{g}^{2}(s) \neq 0$,

$$
\left(\left(\frac{-1}{\kappa_{g}(s)}\right)^{\prime} \frac{1}{\tau_{g}(s)}\right)^{\prime}-\frac{\tau_{g}(s)}{\kappa_{g}(s)}=0
$$


and

$$
\boldsymbol{v}=2 \kappa_{g}^{2}(s) \tau_{g}(s) \sigma^{ \pm}(s) \overline{\boldsymbol{\gamma}}(s)+2 \kappa_{g}(s) \tau_{g}(s) \sigma^{ \pm}(s) \boldsymbol{n}(s)-2 \kappa_{g}^{\prime}(s) \sigma^{ \pm}(s) \boldsymbol{b}(s)+\left(2 \kappa_{g}^{2}(s) \tau_{g}(s) \sigma^{ \pm}(s)+2\right) \boldsymbol{e}_{0} .
$$
$\neq 0$,

(6) $h_{v}(s)=h_{v}^{\prime}(s)=h_{v}^{\prime \prime}(s)=h_{v}^{\prime \prime \prime}(s)=h_{v}^{(4)}(s)=h_{v}^{(5)}(s)=0$ if and only if $\kappa_{g}^{\prime 2}(s)+\kappa_{g}^{2}(s) \tau_{g}^{2}(s)$

$$
\left(\left(\frac{-1}{\kappa_{g}(s)}\right)^{\prime} \frac{1}{\tau_{g}(s)}\right)^{\prime}-\frac{\tau_{g}(s)}{\kappa_{g}(s)}=\left\{\left(\left(\frac{-1}{\kappa_{g}(s)}\right)^{\prime} \frac{1}{\tau_{g}(s)}\right)^{\prime}-\frac{\tau_{g}(s)}{\kappa_{g}(s)}\right\}^{\prime}=0
$$

and

$$
\boldsymbol{v}=2 \kappa_{g}^{2}(s) \tau_{g}(s) \sigma^{ \pm}(s) \overline{\boldsymbol{\gamma}}(s)+2 \kappa_{g}(s) \tau_{g}(s) \sigma^{ \pm}(s) \boldsymbol{n}(s)-2 \kappa_{g}^{\prime}(s) \sigma^{ \pm}(s) \boldsymbol{b}(s)+\left(2 \kappa_{g}^{2}(s) \tau_{g}(s) \sigma^{ \pm}(s)+2\right) \boldsymbol{e}_{0} .
$$

The proof of this proposition is similar to the proof of Proposition 5.1, we omit it. According to the assertions of Propositions 5.1 and 5.2, we define an invariant

$$
\kappa_{S}(s)=\left(\left(\frac{-1}{\kappa_{g}(s)}\right)^{\prime} \frac{1}{\tau_{g}(s)}\right)^{\prime}-\frac{\tau_{g}(s)}{\kappa_{g}(s)} .
$$

We call $\kappa_{S}(s)$ spherical curvature of $\bar{\gamma}$. We have the following proposition.

Proposition 5.3. For a unit speed curve $\gamma: I \rightarrow S_{+}^{3}$, both of the spherical evolutes $\boldsymbol{\varepsilon}_{\bar{\gamma}}^{ \pm}$are single points if and only if $\kappa_{S} \equiv 0$.

Proof.

$$
\begin{gathered}
\kappa_{S}(s)=\left(\frac{\left(-1 / \kappa_{g}(s)\right)^{\prime}}{\tau_{g}(s)}\right)^{\prime}-\frac{\tau_{g}(s)}{\kappa_{g}(s)}=\frac{\kappa_{g}(s) \kappa_{g}^{\prime \prime}(s) \tau_{g}(s)-2 \kappa_{g}^{\prime 2}(s) \tau_{g}(s)-\kappa_{g}(s) \kappa_{g}^{\prime}(s) \tau_{g}^{\prime}(s)-\kappa_{g}^{2}(s) \tau_{g}^{3}(s)}{\kappa_{g}^{3}(s) \tau_{g}^{2}(s)}, \\
\boldsymbol{\varepsilon}_{\bar{\gamma}}^{\prime \pm}(s)=\frac{-\kappa_{S}(s) \kappa_{g}^{4}(s) \tau_{g}^{2}(s) \kappa_{g}^{\prime}(s)}{\left(\kappa_{g}^{\prime 2}(s)+\kappa_{g}^{4}(s) \tau_{g}^{2}(s)+\kappa_{g}^{2}(s) \tau_{g}^{2}(s)\right)^{3 / 2}}\left\{\bar{\gamma}(s)+\frac{1}{\kappa_{g}(s)} \boldsymbol{n}(s)+\frac{\left(1 / \kappa_{g}(s)\right)^{\prime}}{\tau_{g}(s)} \boldsymbol{b}(s)\right\} \\
-\frac{\kappa_{S}(s) \kappa_{g}^{3}(s) \tau_{g}^{2}(s)}{\kappa_{g}(s) \tau_{g}(s)\left(\kappa_{g}^{\prime 2}(s)+\kappa_{g}^{4}(s) \tau_{g}^{2}(s)+\kappa_{g}^{2}(s) \tau_{g}^{2}(s)\right)^{1 / 2}} \boldsymbol{b}(s) .
\end{gathered}
$$

So $\varepsilon_{\bar{\gamma}}^{\prime \pm} \equiv 0$ if and only if $\kappa_{S} \equiv 0$. This completes the proof.

\section{SINGULARITIES OF LIGHTCONE DUALS OF SPHERICAL CURVES}

In this section, we classify the singularities of $\overline{L D}_{\bar{\gamma}}^{ \pm}$and $L D_{\gamma}$ as an application of the unfolding theory of functions. Let $F:\left(\mathbb{R} \times \mathbb{R}^{r},\left(s_{0}, \boldsymbol{x}_{0}\right)\right) \longrightarrow \mathbb{R}$ be a function germ, we call $F$ an $r$-parameter unfolding of $f$, where $f(s)=F_{\boldsymbol{x}_{0}}\left(s, \boldsymbol{x}_{0}\right)$. The discriminant setof $F$ is defined by

$$
D_{F}=\left\{\boldsymbol{x} \in \mathbb{R}^{r} \mid \exists s \in \mathbb{R}, F(s, \boldsymbol{x})=\frac{\partial F}{\partial s}(s, \boldsymbol{x})=0\right\} .
$$

By Propositions 5.1 (2) and 5.2 (2), the discriminant set of $\bar{H}$ and $H$ are

$$
\begin{aligned}
& D_{\bar{H}}=\left\{\bar{\gamma}(s)+u \boldsymbol{n}(s)+v \boldsymbol{b}(s) \pm \sqrt{u^{2}+v^{2}+1} \boldsymbol{e}_{0} \mid s \in I, u, v \in \mathbb{R}\right\}, \\
& D_{H}=\left\{\left(u^{2}+v^{2}-4\right) \bar{\gamma}(s) / 4+u \boldsymbol{n}(s)+v \boldsymbol{b}(s)+\left(u^{2}+v^{2}+4\right) \boldsymbol{e}_{0} / 4 \mid s \in I, u, v \in \mathbb{R}\right\} .
\end{aligned}
$$


These are the lightcone dual hypersurfaces of $\bar{\gamma}$ and the lightcone dual surface of $\boldsymbol{\gamma}$, respectively. Moreover, the both assertions (4) of Propositions 5.1 and 5.2 describe the singularities of the lightcone focal surfaces of $\boldsymbol{\gamma}$ and $\bar{\gamma}$, respectively.

Proposition 6.1. The critical value sets of $L F_{\bar{\gamma}}^{ \pm}$and $L F_{\gamma}^{ \pm}$are give as follows:

$$
\begin{aligned}
& C\left(L F_{\bar{\gamma}}^{ \pm}\right)=\left\{\bar{\gamma}(s)+\frac{1}{\kappa_{g}(s)} \boldsymbol{n}(s)-\frac{\kappa_{g}^{\prime}(s)}{\kappa_{g}^{2}(s) \tau_{g}(s)} \boldsymbol{b}(s) \pm \rho(s) \boldsymbol{e}_{0} \mid s \in I\right\}, \\
& C\left(L F_{\gamma}^{ \pm}\right)=\left\{2 \kappa_{g}^{2}(s) \tau_{g}(s) \sigma^{ \pm}(s) \bar{\gamma}(s)+2 \kappa_{g}(s) \tau_{g}(s) \sigma^{ \pm}(s) \boldsymbol{n}(s)\right. \\
&\left.\quad-2 \kappa_{g}^{\prime}(s) \sigma^{ \pm}(s) \boldsymbol{b}(s)+\left(2 \kappa_{g}^{2}(s) \tau_{g}(s) \sigma^{ \pm}(s)+2\right) \boldsymbol{e}_{0} \mid s \in I\right\} .
\end{aligned}
$$

Then we have the following theorem as a corollary.

Theorem 6.2. Both of the projections of the critical value sets $C\left(L F_{\bar{\gamma}}^{ \pm}\right)$and $C\left(L F_{\gamma}^{ \pm}\right)$in the unit 3-sphere $S_{0}^{3}$ are the images of the spherical evolutes of $\bar{\gamma}$, that is,

$$
\tilde{\pi}\left(C\left(L F_{\bar{\gamma}}^{ \pm}\right)\right)=\tilde{\pi}\left(C\left(L F_{\gamma}^{ \pm}\right)\right)=\left\{\varepsilon_{\bar{\gamma}}^{ \pm}(s) \mid s \in I\right\} .
$$

Proof. We know that

$$
\tilde{\pi}\left(C\left(L F_{\bar{\gamma}}^{ \pm}\right)\right)=\left\{ \pm \frac{1}{\rho(s)}\left(\overline{\boldsymbol{\gamma}}(s)+\frac{1}{\kappa_{g}(s)} \boldsymbol{n}(s)-\frac{\boldsymbol{\kappa}_{g}^{\prime}(s)}{\boldsymbol{\kappa}_{g}^{2}(s) \tau_{g}(s)} \boldsymbol{b}(s)\right) \mid s \in I\right\}
$$

and

$$
\tilde{\pi}\left(C\left(L F_{\gamma}^{ \pm}\right)\right)=\left\{\sigma^{ \pm}(s) \frac{\kappa_{g}^{2}(s) \tau_{g}(s) \bar{\gamma}(s)+\kappa_{g}(s) \tau_{g}(s) \boldsymbol{n}(s)-\kappa_{g}^{\prime}(s) \boldsymbol{b}(s)}{\kappa_{g}^{2}(s) \tau_{g}(s) \sigma^{ \pm}(s)+1} \mid s \in I\right\} .
$$

By straightforward calculations, we have

$$
\begin{aligned}
& \frac{\kappa_{g}^{2}(s) \tau_{g}(s) \sigma^{ \pm}(s)}{\kappa_{g}^{2}(s) \tau_{g}(s) \sigma^{ \pm}(s)+1} \\
= & \frac{\kappa_{g}^{2}(s) \tau_{g}(s)\left(\kappa_{g}^{2}(s) \tau_{g}(s) \pm \sqrt{\kappa_{g}^{\prime 2}(s)+\kappa_{g}^{2}(s) \tau_{g}^{2}(s)+\kappa_{g}^{4}(s) \tau_{g}^{2}(s)}\right)}{\kappa_{g}^{\prime 2}(s)+\kappa_{g}^{2}(s) \tau_{g}^{2}(s)+\kappa_{g}^{4}(s) \tau_{g}^{2}(s) \pm \kappa_{g}^{2}(s) \tau_{g}(s) \sqrt{\kappa_{g}^{\prime 2}(s)+\kappa_{g}^{2}(s) \tau_{g}^{2}(s)+\kappa_{g}^{4}(s) \tau_{g}^{2}(s)}} \\
= & \frac{ \pm \kappa_{g}^{2}(s) \tau_{g}(s)}{\sqrt{\kappa_{g}^{\prime 2}(s)+\kappa_{g}^{2}(s) \tau_{g}^{2}(s)+\kappa_{g}^{4}(s) \tau_{g}^{2}(s)}}=\frac{ \pm 1}{\rho(s)} .
\end{aligned}
$$

Similarly, we can calculate that

$$
\begin{gathered}
\frac{\kappa_{g}(s) \tau_{g}(s) \sigma^{ \pm}(s)}{\kappa_{g}^{2}(s) \tau_{g}(s) \sigma^{ \pm}(s)+1}=\frac{ \pm 1}{\rho(s) \kappa_{g}(s)}, \\
\frac{\kappa_{g}^{\prime}(s) \sigma^{ \pm}(s)}{\kappa_{g}^{2}(s) \tau_{g}(s) \sigma^{ \pm}(s)+1}=\frac{ \pm \kappa_{g}^{\prime}(s)}{\rho(s) \kappa_{g}^{2}(s) \tau_{g}(s)} .
\end{gathered}
$$

Thus we have

$$
\tilde{\pi}\left(C\left(L F_{\bar{\gamma}}^{ \pm}\right)\right)=\tilde{\pi}\left(C\left(L F_{\gamma}^{ \pm}\right)\right)=\left\{\varepsilon_{\bar{\gamma}}^{ \pm}(s) \mid s \in I\right\} .
$$

This completes the proof. 
Inspired by Propositons 5.1, 5.2, and Theorem 6.2, we define the following set:

$$
D_{F}^{\ell}=\left\{\boldsymbol{x} \in \mathbb{R}^{r} \mid \exists s \in \mathbb{R}, F(s, \boldsymbol{x})=\frac{\partial F}{\partial s}(s, \boldsymbol{x})=\cdots=\frac{\partial^{\ell} F}{\partial s^{\ell}}(s, \boldsymbol{x})=0\right\},
$$

which we call the discriminant set of order $\ell$. Of course, $D_{F}^{1}=D_{F}$. In order to understand the geometric properties of the discriminant set of order $\ell$, we introduce an equivalence relation among the unfoldings of functions. Let $F$ and $G$ be $r$-parameter unfoldings of $f(s)$ and $g(s)$, respectively. We say that $F$ and $G$ are $P$-R -equivalent if there exists a diffeomorphism germ $\Phi:\left(\mathbb{R} \times \mathbb{R}^{r},\left(s_{0}, \boldsymbol{x}_{0}\right)\right) \longrightarrow\left(\mathbb{R} \times \mathbb{R}^{r},\left(s_{0}^{\prime}, \boldsymbol{x}_{0}^{\prime}\right)\right)$ of the form $\Phi(s, \boldsymbol{x})=\left(\Phi_{1}(s, \boldsymbol{x}), \phi(\boldsymbol{x})\right)$ such that $G \circ \Phi=F$. By straightforward calculations, we have the following proposition.

Proposition 6.3. Let $F$ and $G$ be r-parameter unfoldings of $f(s)$ and $g(s)$, respectively. If $F$ and $G$ are $P$ - $\mathcal{R}$-equivalent by a diffeomorphism germ $\Phi:\left(\mathbb{R} \times \mathbb{R}^{r},\left(s_{0}, \boldsymbol{x}_{0}\right)\right) \longrightarrow\left(\mathbb{R} \times \mathbb{R}^{r},\left(s_{0}^{\prime}, \boldsymbol{x}_{0}^{\prime}\right)\right)$ of the form $\Phi(s, \boldsymbol{x})=\left(\Phi_{1}(s, \boldsymbol{x}), \phi(\boldsymbol{x})\right)$, then $\phi\left(D_{F}^{\ell}\right)=D_{G}^{\ell}$ as set germs.

By Propositions 5.1 and 5.2, we have the following proposition.

Proposition 6.4. Under the same notations as in the previous paragraphs, we have

$$
\begin{aligned}
& D_{\bar{H}}=D_{\bar{H}}^{1}=\text { Images } \overline{L D_{\bar{\gamma}}^{ \pm}}, D_{\bar{H}}^{2}=\operatorname{Images} L F_{\bar{\gamma}}^{ \pm}, \tilde{\pi}\left(D_{\bar{H}}^{3}\right)=\operatorname{Images} \boldsymbol{\varepsilon}_{\bar{\gamma}}^{ \pm}, \\
& D_{H}=D_{H}^{1}=\text { Image } L D_{\gamma}, D_{H}^{2}=\text { Images } L F_{\gamma}^{ \pm}, \tilde{\pi}\left(D_{H}^{3}\right)=\operatorname{Images} \boldsymbol{\varepsilon}_{\bar{\gamma}}^{ \pm} .
\end{aligned}
$$

Let $F$ be an $r$-parameter unfolding of $f$ and $f$ has $A_{k}$-singularity $(k \geq 1)$ at $s_{0}$. We denote the $(k-1)$-jet of the partial derivative $\partial F / \partial x_{i}$ at $s_{0}$ as

$$
j^{(k-1)}\left(\frac{\partial F}{\partial x_{i}}\left(s, \boldsymbol{x}_{0}\right)\right)\left(s_{0}\right)=\sum_{j=1}^{k-1} \alpha_{j i}\left(s-s_{0}\right)^{j}, i=1, \cdots, r .
$$

If the rank of $k \times r$ matrix $\left(\alpha_{0 i}, \alpha_{j i}\right)$ is $k(k \leq r)$, then $F$ is called a versal unfolding of $f$, where $\alpha_{0 i}=\partial F / \partial x_{i}\left(s_{0}, \boldsymbol{x}_{0}\right)$. We have the following classification theorem of versal unfoldings (see page 149, Sec. 6.6 of Ref. 3).

Theorem 6.5. Let $F:\left(\mathbb{R} \times \mathbb{R}^{r},\left(s_{0}, \boldsymbol{x}_{0}\right)\right) \longrightarrow \mathbb{R}$ be an r-parameter unfolding of $f$ which has $A_{k}$-singularity at $s_{0}$. Suppose $F$ is a versal unfolding of $f$, then $F$ is $P$ - $\mathcal{R}$-equivalent to one of the following unfoldings:

(a) $k=1: \pm s^{2}+x_{1}$,

(b) $k=2: s^{3}+x_{1}+s x_{2}$,

(c) $k=3: \pm s^{4}+x_{1}+s x_{2}+s^{2} x_{3}$,

(d) $k=4: s^{5}+x_{1}+s x_{2}+s^{2} x_{3}+s^{3} x_{4}$.

We have the following classification result as a corollary of the above theorem.

Corollary 6.6. Let $F:\left(\mathbb{R} \times \mathbb{R}^{r},\left(s_{0}, \boldsymbol{x}_{0}\right)\right) \longrightarrow \mathbb{R}$ be an r-parameter unfolding of $f$ which has $A_{k}$-singularity at $s_{0}$. Suppose $F$ is a versal unfolding of $f$, then we have the following assertions:

(a) For $k=1, D_{F}$ is diffeomorphic to $\{0\} \times \mathbb{R}^{r-1}$ and $D_{F}^{2}=\emptyset$.

(b) For $k=2, D_{F}$ is diffeomorphic to $C(2,3) \times \mathbb{R}^{r-2}, D_{F}^{2}$ is diffeomorphic to $\{\mathbf{0}\} \times \mathbb{R}^{r-2}$ and $D_{F}^{3}=\varnothing$.

(c) For $k=3, D_{F}$ is diffeomorphic to $S W \times \mathbb{R}^{r-3}, D_{F}^{2}$ is diffeomorphic to $C(2,3,4) \times \mathbb{R}^{r-3}, D_{F}^{3}$ is diffeomorphic to $\{\mathbf{0}\} \times \mathbb{R}^{r-3}$ and $D_{F}^{4}=\emptyset$.

(d) For $k=4, D_{F}$ is locally diffeomorphic to $B F \times \mathbb{R}^{r-4}, D_{F}^{2}$ is diffeomorphic to $C(B F)$ $\times \mathbb{R}^{r-4}, D_{F}^{3}$ is diffeomorphic to $C(2,3,4,5) \times \mathbb{R}^{r-4}, D_{F}^{4}$ is diffeomorphic to $\{\mathbf{0}\} \times \mathbb{R}^{r-4}$ and $D_{F}^{5}=\emptyset$.

We remark that all of diffeomorphisms in the above assertions are diffeomorphism germs. 
Here, we respectively call

$$
C(2,3)=\left\{\left(x_{1}, x_{2}\right) \mid x_{1}=u^{2}, x_{2}=u^{3}\right\}
$$

a (2, 3)-cusp,

$$
C(2,3,4)=\left\{\left(x_{1}, x_{2}, x_{3}\right) \mid x_{1}=u^{2}, x_{2}=u^{3}, x_{3}=u^{4}\right\}
$$

a $(2,3,4)-c u s p$,

$$
C(2,3,4,5)=\left\{\left(x_{1}, x_{2}, x_{3}, x_{4}\right) \mid x_{1}=u^{2}, x_{2}=u^{3}, x_{3}=u^{4}, x_{4}=u^{5}\right\}
$$

a $(2,3,4,5)$-cusp,

$$
S W=\left\{\left(x_{1}, x_{2}, x_{3}\right) \mid x_{1}=3 u^{4}+u^{2} v, x_{2}=4 u^{3}+2 u v, x_{3}=v\right\}
$$

a swallow tail,

$$
B F=\left\{\left(x_{1}, x_{2}, x_{3} . x_{4}\right) \mid x_{1}=5 u^{4}+3 v u^{2}+2 w u, x_{2}=4 u^{5}+2 v u^{3}+w u^{2}, x_{3}=u, x_{4}=v\right\}
$$

a butterfly,

$$
C(B F)=\left\{\left(x_{1}, x_{2}, x_{3}, x_{4}\right) \mid x_{1}=6 u^{5}+u^{3} v, x_{2}=25 u^{4}+9 u^{2} v, x_{3}=10 u^{3}+3 u v, x_{4}=v\right\}
$$

a $c$-butterfly (i.e., the critical value set of the butterfly).

We have the following key propositions for $H$ and $\bar{H}$. of $\bar{h}_{v_{0}}$.

Proposition 6.7. If $\bar{h}_{v_{0}}$ has an $A_{k}$-singularity, $k=1,2,3,4$, at $s_{0}$, then $\bar{H}$ is a versal unfolding

Proof. For $\boldsymbol{v} \in L C^{*}$, we have $\boldsymbol{v}=\left( \pm\left(v_{1}^{2}+v_{2}^{2}+v_{3}^{2}+v_{4}^{2}\right)^{1 / 2}, v_{1}, v_{2}, v_{3}, v_{4}\right)$. We denote that $\bar{\gamma}(s)=\left(0, x_{1}(s), x_{2}(s), x_{3},(s), x_{4}(s)\right)$. Then

$$
\bar{H}(s, \boldsymbol{v})=\langle\bar{\gamma}(s), \boldsymbol{v}\rangle-1=x_{1}(s) v_{1}+x_{2}(s) v_{2}+x_{3}(s) v_{3}+x_{4}(s) v_{4}-1 .
$$

Thus we have

$$
\begin{gathered}
\frac{\partial \bar{H}}{\partial v_{1}}(s, \boldsymbol{v})=x_{1}(s), \frac{\partial \bar{H}}{\partial v_{2}}(s, \boldsymbol{v})=x_{2}(s), \frac{\partial \bar{H}}{\partial v_{3}}(s, \boldsymbol{v})=x_{3}(s), \frac{\partial \bar{H}}{\partial v_{4}}(s, \boldsymbol{v})=x_{4}(s), \\
\frac{\partial^{2} \bar{H}}{\partial s \partial v_{1}}(s, \boldsymbol{v})=x_{1}^{\prime}(s), \frac{\partial^{2} \bar{H}}{\partial s \partial v_{2}}(s, \boldsymbol{v})=x_{2}^{\prime}(s), \frac{\partial^{2} \bar{H}}{\partial s \partial v_{3}}(s, \boldsymbol{v})=x_{3}^{\prime}(s), \frac{\partial^{2} \bar{H}}{\partial s \partial v_{4}}(s, \boldsymbol{v})=x_{4}^{\prime}(s), \\
\frac{\partial^{3} \bar{H}}{\partial s^{2} \partial v_{1}}(s, \boldsymbol{v})=x_{1}^{\prime \prime}(s), \frac{\partial^{3} \bar{H}}{\partial s^{2} \partial v_{2}}(s, \boldsymbol{v})=x_{2}^{\prime \prime}(s), \frac{\partial^{3} \bar{H}}{\partial s^{2} \partial v_{3}}(s, \boldsymbol{v})=x_{3}^{\prime \prime}(s), \frac{\partial^{3} \bar{H}}{\partial s^{2} \partial v_{4}}(s, \boldsymbol{v})=x_{4}^{\prime \prime}(s), \\
\frac{\partial^{4} \bar{H}}{\partial s^{3} \partial v_{1}}(s, \boldsymbol{v})=x_{1}^{\prime \prime \prime}(s), \frac{\partial^{4} \bar{H}}{\partial s^{3} \partial v_{2}}(s, \boldsymbol{v})=x_{2}^{\prime \prime \prime}(s), \frac{\partial^{4} \bar{H}}{\partial s^{3} \partial v_{3}}(s, \boldsymbol{v})=x_{3}^{\prime \prime \prime}(s), \frac{\partial^{4} \bar{H}}{\partial s^{3} \partial v_{4}}(s, \boldsymbol{v})=x_{4}^{\prime \prime \prime}(s) .
\end{gathered}
$$

For a fixed point $\boldsymbol{v}_{0}=\left(v_{00}, v_{01}, v_{02}, v_{03}, v_{04}\right)$, the 3 -jet of $\partial \bar{H} / \partial v_{i}\left(s, \boldsymbol{v}_{0}\right)$ at $s_{0}$ is

$$
j^{(3)} \frac{\partial \bar{H}}{\partial v_{i}}\left(s, v_{0}\right)\left(s_{0}\right)=x_{i}^{\prime}\left(s_{0}\right)\left(s-s_{0}\right)+x_{i}^{\prime \prime}\left(s_{0}\right)\left(s-s_{0}\right)^{2} / 2+x_{i}^{\prime \prime \prime}\left(s_{0}\right)\left(s-s_{0}\right)^{3} / 6, i=1,2,3,4 \text {. }
$$

For $k=1,2,3$, it is obviously that $\bar{H}$ is a versal unfolding of $\bar{h}_{v_{0}}$. So we only consider the case $k=4$. We need to show that the rank of the matrix $\mathrm{A}$ is 4 , where

$$
A=\left(\begin{array}{cccc}
x_{1}\left(s_{0}\right) & x_{2}\left(s_{0}\right) & x_{3}\left(s_{0}\right) & x_{4}\left(s_{0}\right) \\
x_{1}^{\prime}\left(s_{0}\right) & x_{2}^{\prime}\left(s_{0}\right) & x_{3}^{\prime}\left(s_{0}\right) & x_{4}^{\prime}\left(s_{0}\right) \\
x_{1}^{\prime \prime}\left(s_{0}\right) & x_{2}^{\prime \prime}\left(s_{0}\right) & x_{3}^{\prime \prime}\left(s_{0}\right) & x_{4}^{\prime \prime}\left(s_{0}\right) \\
x_{1}^{\prime \prime \prime}\left(s_{0}\right) & x_{2}^{\prime \prime \prime}\left(s_{0}\right) & x_{3}^{\prime \prime \prime}\left(s_{0}\right) & x_{4}^{\prime \prime \prime}\left(s_{0}\right)
\end{array}\right) .
$$


We have

$$
\operatorname{det} A=\left\langle\boldsymbol{e}_{0} \wedge \overline{\boldsymbol{\gamma}}\left(s_{0}\right) \wedge \overline{\boldsymbol{\gamma}}^{\prime}\left(s_{0}\right) \wedge \overline{\boldsymbol{\gamma}}^{\prime \prime}\left(s_{0}\right), \overline{\boldsymbol{\gamma}}^{\prime \prime \prime}\left(s_{0}\right)\right\rangle=-\kappa_{g}^{2}\left(s_{0}\right) \tau_{g}\left(s_{0}\right) \neq 0,
$$

which completes the proof. of $h_{v_{0}}$

Proposition 6.8. If $h_{v_{0}}$ has an $A_{k}$-singularity, $k=1,2,3,4$, at $s_{0}$, then $H$ is a versal unfolding

Proof. For $\quad \boldsymbol{v} \in L C^{*}, \quad$ we $\quad$ have $\quad \boldsymbol{v}=\left(v_{0}, v_{1}, v_{2}, v_{3}, v_{4}\right)=\left( \pm\left(v_{1}^{2}+v_{2}^{2}+v_{3}^{2}\right.\right.$ $\left.\left.+v_{4}^{2}\right)^{1 / 2}, v_{1}, v_{2}, v_{3}, v_{4}\right)$. We write $\bar{\gamma}(s)=\left(1, x_{1}(s), x_{2}(s), x_{3}(s), x_{4}(s)\right)$. Then we have $H(s, \boldsymbol{v})=\langle\boldsymbol{\gamma}(s), \boldsymbol{v}\rangle+2=\mp\left(v_{1}^{2}+v_{2}^{2}+v_{3}^{2}+v_{4}^{2}\right)^{1 / 2}+x_{1}(s) v_{1}+x_{2}(s) v_{2}+x_{3}(s) v_{3}+x_{4}(s) v_{4}+2$.

Thus we have

$$
\begin{gathered}
\frac{\partial H}{\partial v_{1}}(s, \boldsymbol{v})=-v_{1} / v_{0}+x_{1}(s), \frac{\partial H}{\partial v_{2}}(s, \boldsymbol{v})=-v_{2} / v_{0}+x_{2}(s), \\
\frac{\partial H}{\partial v_{3}}(s, \boldsymbol{v})=-v_{3} / v_{0}+x_{3}(s), \frac{\partial H}{\partial v_{4}}(s, \boldsymbol{v})=-v_{4} / v_{0}+x_{4}(s), \\
\frac{\partial^{2} H}{\partial s \partial v_{1}}(s, \boldsymbol{v})=x_{1}^{\prime}(s), \frac{\partial^{2} H}{\partial s \partial v_{2}}(s, \boldsymbol{v})=x_{2}^{\prime}(s), \frac{\partial^{2} H}{\partial s \partial v_{3}}(s, \boldsymbol{v})=x_{3}^{\prime}(s), \frac{\partial^{2} H}{\partial s \partial v_{4}}(s, \boldsymbol{v})=x_{4}^{\prime}(s), \\
\frac{\partial^{3} H}{\partial s^{2} \partial v_{1}}(s, \boldsymbol{v})=x_{1}^{\prime \prime}(s), \frac{\partial^{3} H}{\partial s^{2} \partial v_{2}}(s, \boldsymbol{v})=x_{2}^{\prime \prime}(s), \frac{\partial^{3} H}{\partial s^{2} \partial v_{3}}(s, \boldsymbol{v})=x_{3}^{\prime \prime}(s), \frac{\partial^{3} H}{\partial s^{2} \partial v_{4}}(s, \boldsymbol{v})=x_{4}^{\prime \prime}(s), \\
\frac{\partial^{4} H}{\partial s^{3} \partial v_{1}}(s, \boldsymbol{v})=x_{1}^{\prime \prime \prime}(s), \frac{\partial^{4} H}{\partial s^{3} \partial v_{2}}(s, \boldsymbol{v})=x_{2}^{\prime \prime \prime}(s), \frac{\partial^{4} H}{\partial s^{3} \partial v_{3}}(s, \boldsymbol{v})=x_{3}^{\prime \prime \prime}(s), \frac{\partial^{4} H}{\partial s^{3} \partial v_{4}}(s, \boldsymbol{v})=x_{4}^{\prime \prime \prime}(s) .
\end{gathered}
$$

For a fixed $\boldsymbol{v}_{0}=\left(v_{00}, v_{01}, v_{02}, v_{03}, v_{04}\right)$, the 3-jet of $\partial H / \partial v_{i}\left(s, \boldsymbol{v}_{0}\right)$ at $s_{0}$ is

$$
j^{(3)} \frac{\partial H}{\partial v_{i}}\left(s, \boldsymbol{v}_{0}\right)\left(s_{0}\right)=x_{i}^{\prime}\left(s_{0}\right)\left(s-s_{0}\right)+x_{i}^{\prime \prime}\left(s_{0}\right)\left(s-s_{0}\right)^{2} / 2+x_{i}^{\prime \prime \prime}\left(s_{0}\right)\left(s-s_{0}\right)^{3} / 6, i=1,2,3,4 .
$$

For $k=1,2,3$, it is obviously that $H$ is a versal unfolding of $h_{v_{0}}$. So we only consider the case $k=4$. We need to show that the rank of the matrix $\mathrm{B}$ is 4 , where

$$
B=\left(\begin{array}{cccc}
-v_{01} / v_{00}+x_{1}\left(s_{0}\right) & -v_{02} / v_{00}+x_{2}\left(s_{0}\right) & -v_{03} / v_{00}+x_{3}\left(s_{0}\right) & -v_{04} / v_{00}+x_{4}\left(s_{0}\right) \\
x_{1}^{\prime}\left(s_{0}\right) & x_{2}^{\prime}\left(s_{0}\right) & x_{3}^{\prime}\left(s_{0}\right) & x_{4}^{\prime}\left(s_{0}\right) \\
x_{1}^{\prime \prime}\left(s_{0}\right) & x_{2}^{\prime \prime}\left(s_{0}\right) & x_{3}^{\prime \prime}\left(s_{0}\right) & x_{4}^{\prime \prime}\left(s_{0}\right) \\
x_{1}^{\prime \prime \prime}\left(s_{0}\right) & x_{2}^{\prime \prime \prime}\left(s_{0}\right) & x_{3}^{\prime \prime \prime}\left(s_{0}\right) & x_{4}^{\prime \prime \prime}\left(s_{0}\right)
\end{array}\right) .
$$

By straightforward calculations, we have

$$
\operatorname{det} B=\left\langle\boldsymbol{e}_{0} \wedge \overline{\boldsymbol{\gamma}}^{\prime}\left(s_{0}\right) \wedge \overline{\boldsymbol{\gamma}}^{\prime \prime}\left(s_{0}\right) \wedge \overline{\boldsymbol{\gamma}}^{\prime \prime \prime}\left(s_{0}\right), \boldsymbol{v}_{0}\right\rangle / v_{00}+\left\langle\boldsymbol{e}_{0} \wedge \overline{\boldsymbol{\gamma}}\left(s_{0}\right) \wedge \overline{\boldsymbol{\gamma}}^{\prime}\left(s_{0}\right) \wedge \overline{\boldsymbol{\gamma}}^{\prime \prime}\left(s_{0}\right), \overline{\boldsymbol{\gamma}}^{\prime \prime \prime}\left(s_{0}\right)\right\rangle
$$

$=\left\langle\kappa_{g}^{2}\left(s_{0}\right) \tau_{g}\left(s_{0}\right) \overline{\boldsymbol{\gamma}}\left(s_{0}\right), \boldsymbol{v}_{0}\right\rangle / v_{00}-\left\langle\kappa_{g}^{\prime}\left(s_{0}\right) \boldsymbol{b}\left(s_{0}\right), \boldsymbol{v}_{0}\right\rangle / v_{00}+\left\langle\kappa_{g}\left(s_{0}\right) \tau_{g}\left(s_{0}\right) \boldsymbol{n}\left(s_{0}\right), \boldsymbol{v}_{0}\right\rangle / v_{00}-\kappa_{g}^{2}\left(s_{0}\right) \tau_{g}\left(s_{0}\right)$.

In this case, $h_{v_{0}}(s)$ has an $A_{4}$-singularity, then we have

$$
\begin{gathered}
\boldsymbol{v}_{0}=2 \kappa_{g}^{2}\left(s_{0}\right) \tau_{g}\left(s_{0}\right) \sigma^{ \pm}\left(s_{0}\right) \overline{\boldsymbol{\gamma}}\left(s_{0}\right)+2 \kappa_{g}\left(s_{0}\right) \tau_{g}\left(s_{0}\right) \sigma^{ \pm}\left(s_{0}\right) \boldsymbol{n}\left(s_{0}\right)-2 \kappa_{g}^{\prime}\left(s_{0}\right) \sigma^{ \pm}\left(s_{0}\right) \boldsymbol{b}\left(s_{0}\right) \\
+\left(2 \kappa_{g}^{2}\left(s_{0}\right) \tau_{g}\left(s_{0}\right) \sigma^{ \pm}\left(s_{0}\right)+2\right) \boldsymbol{e}_{0} .
\end{gathered}
$$


Moreover, we have

$$
v_{00}=2 \kappa_{g}^{2}\left(s_{0}\right) \tau_{g}\left(s_{0}\right) \sigma^{ \pm}\left(s_{0}\right)+2 .
$$

Therefore by calculation, we have

$$
\operatorname{det} B= \pm \frac{\kappa_{g}^{2}\left(s_{0}\right) \tau_{g}^{2}\left(s_{0}\right)+\kappa_{g}^{\prime 2}\left(s_{0}\right)}{\sqrt{\kappa_{g}^{\prime 2}\left(s_{0}\right)+\kappa_{g}^{2}\left(s_{0}\right) \tau_{g}^{2}\left(s_{0}\right)+\kappa_{g}^{4}\left(s_{0}\right) \tau_{g}^{2}\left(s_{0}\right)} \pm \kappa_{g}^{2}\left(s_{0}\right) \tau_{g}\left(s_{0}\right)} \neq 0,
$$

which completes the proof.

We have the following theorem.

Theorem 6.9. Let $\gamma: I \longrightarrow S_{+}^{3}$ be a unit speed curve.

(A) For the lightcone duals $\overline{L D}_{\bar{\gamma}}^{ \pm}$of $\bar{\gamma}$, we have the following assertions:

(1) Each one of the lightcone duals $\overline{L D}_{\bar{\gamma}}^{ \pm}$of $\bar{\gamma}$ is locally diffeomorphic to $C(2,3) \times \mathbb{R}^{2}$ at $\left(s_{0}, u_{0}, v_{0}\right)$ if and only if

$$
\kappa_{g}\left(s_{0}\right) \neq 0, u_{0}=\frac{1}{\kappa_{g}\left(s_{0}\right)} \text { and } v_{0} \neq\left(\frac{1}{\kappa_{g}\left(s_{0}\right)}\right)^{\prime} \frac{1}{\tau_{g}\left(s_{0}\right)} .
$$

In this case, each one of $L F_{\bar{\gamma}}^{ \pm}$is non-singular and each one of Images $\boldsymbol{\varepsilon}_{\bar{\gamma}}^{ \pm}$is empty.

(2) Each one of the lightcone duals $\overline{L D_{\bar{\gamma}}^{ \pm}}$of $\bar{\gamma}$ is locally diffeomorphic to $S W \times \mathbb{R}$ at $\left(s_{0}, u_{0}, v_{0}\right)$ if and only if

$$
\kappa_{g}\left(s_{0}\right) \neq 0, \tau_{g}\left(s_{0}\right) \neq 0, u_{0}=\frac{1}{\kappa_{g}\left(s_{0}\right)}, v_{0}=\left(\frac{1}{\kappa_{g}\left(s_{0}\right)}\right)^{\prime} \frac{1}{\tau_{g}\left(s_{0}\right)}, \text { and } \kappa_{S}\left(s_{0}\right) \neq 0 .
$$

In this case, each one of $L F_{\bar{\gamma}}^{ \pm}$is locally diffeomorphic to $C(2,3,4) \times \mathbb{R}$ and each one of Images $\boldsymbol{\varepsilon}_{\bar{\gamma}}^{ \pm}$ is a regular curve.

(3) Each one of the lightcone duals $\overline{L D}_{\bar{\gamma}}^{ \pm}$of $\bar{\gamma}$ is locally diffeomorphic to BF at $\left(s_{0}, u_{0}, v_{0}\right)$ if and only if

$$
\kappa_{g}\left(s_{0}\right) \neq 0, \tau_{g}\left(s_{0}\right) \neq 0, u_{0}=\frac{1}{\kappa_{g}\left(s_{0}\right)}, v_{0}=\left(\frac{1}{\kappa_{g}\left(s_{0}\right)}\right)^{\prime} \frac{1}{\tau_{g}\left(s_{0}\right)}, \kappa_{S}\left(s_{0}\right)=0, \text { and } \kappa_{S}^{\prime}\left(s_{0}\right) \neq 0 .
$$

In this case, each one of $L F_{\bar{\gamma}}^{ \pm}$is locally diffeomorphic to $C(B F) \times \mathbb{R}$ and each one of Images $\varepsilon_{\bar{\gamma}}^{ \pm}$is locally diffeomorphic to the projection of the $C(2,3,4,5)$ to $S_{0}^{3}$.

(B) For the lightcone dual $L D_{\gamma}$ of $\gamma$, we have the following assertions:

(1) The lightcone dual $L D_{\gamma}$ of $\gamma$ is locally diffeomorphic to $C(2,3) \times \mathbb{R}^{2}$ at $\left(s_{0}, u_{0}, v_{0}\right)$ if and only if

$$
u_{0} \neq 2 \kappa_{g}\left(s_{0}\right) \tau_{g}\left(s_{0}\right) \sigma^{ \pm}\left(s_{0}\right) \text { and } v_{0}= \pm \sqrt{4+4 \kappa_{g}\left(s_{0}\right) u_{0}-u_{0}^{2}} .
$$

In this case, each one of $L F_{\gamma}^{ \pm}$is non-singular and each one of Images $\boldsymbol{\varepsilon}_{\bar{\gamma}}^{ \pm}$is empty.

(2) The lightcone dual $L D_{\gamma}$ of $\gamma$ is locally diffeomorphic to $S W \times \mathbb{R}$ at $\left(s_{0}, u_{0}, v_{0}\right)$ if and only if

$$
\kappa_{g}^{\prime 2}\left(s_{0}\right)+\kappa_{g}^{2}\left(s_{0}\right) \tau_{g}^{2}\left(s_{0}\right) \neq 0, u_{0}=2 \kappa_{g}\left(s_{0}\right) \tau_{g}\left(s_{0}\right) \sigma^{ \pm}\left(s_{0}\right), v_{0}=-2 \kappa_{g}^{\prime}\left(s_{0}\right) \sigma^{ \pm}\left(s_{0}\right), \text { and } \kappa_{S}\left(s_{0}\right) \neq 0 .
$$

In this case, each one of $L F_{\gamma}^{ \pm}$is locally diffeomorphic to $C(2,3,4) \times \mathbb{R}$ and each one of Images $\varepsilon_{\bar{\gamma}}^{ \pm}$ is a regular curve.

(3) The lightcone dual $L D_{\gamma}$ of $\gamma$ is locally diffeomorphic to BF at $\left(s_{0}, u_{0}, v_{0}\right)$ if and only if

$$
\begin{gathered}
\kappa_{g}^{\prime 2}\left(s_{0}\right)+\kappa_{g}^{2}\left(s_{0}\right) \tau_{g}^{2}\left(s_{0}\right) \neq 0, u_{0}=2 \kappa_{g}\left(s_{0}\right) \tau_{g}\left(s_{0}\right) \sigma^{ \pm}\left(s_{0}\right), v_{0}=-2 \kappa_{g}^{\prime}\left(s_{0}\right) \sigma^{ \pm}\left(s_{0}\right), \\
\kappa_{S}\left(s_{0}\right)=0 \text { and } \kappa_{S}^{\prime}\left(s_{0}\right) \neq 0 .
\end{gathered}
$$


In this case, each one of $L F_{\gamma}^{ \pm}$is locally diffeomorphic to $C(B F) \times \mathbb{R}$ and each one of Images $\boldsymbol{\varepsilon}_{\bar{\gamma}}^{ \pm}$is locally diffeomorphic to the projection of the $C(2,3,4,5)$-cusp to $S_{0}^{3}$.

Proof. By Propositions 5.1 and 5.2, the discriminant sets of $\bar{H}$ and $H$ are the lightcone duals of $\bar{\gamma}$ and $\boldsymbol{\gamma}$, respectively. By Propositions 5.1 and 5.2, both of $\bar{h}_{v_{0}}$ and $h_{v_{0}}$ have $A_{k}$ singularities $(k=$ $1,2,3,4)$, respectively, if and only if the above conditions on the geodesic curvatures and geodesic torsions hold. By Propositions 6.7 and 6.8, $\bar{H}$ and $H$ are versal unfoldings of $\bar{h}_{v_{0}}$ and $h_{v_{0}}$ at any point $s_{0} \in I$, respectively. We apply Corollary 6.6, so that we have the above assertions. This completes the proof.

\section{ACKNOWLEDGMENTS}

We record here our warmest acknowledgment to Professor Donghe Pei. He has given us many precious suggestions during the writing of this paper. The corresponding author Yang Jiang also acknowledges the support from National Science Foundation (NSF) of China No. 11271063 and NCET of China No. 05-0319.

${ }^{1}$ V. I. Arnol'd, S. M. Gusein-Zade, and A. N. Varchenko, Singularities of Differentiable Maps (Birkhäuser, 1986), Vol. I.

${ }^{2}$ J. W. Bruce and M. C. Romero-Fuster, "Duality and projections of curves and surfaces in 3-space," Q. J. Math. Oxford Ser. 42, 433-441 (1991).

${ }^{3}$ J. W. Bruce and P. J. Giblin, Curves and Singularities, 2nd ed. (Cambridge University Press, 1992).

${ }^{4}$ L. Chen and S. Izumiya, "A mandala of Legendrian dualities for pseudo-spheres in semi-Euclidean space," Proc. Jpn. Acad., Ser. A: Math. Sci. 85, 49-54 (2009).

${ }^{5}$ S. Izumiya, "Lengendrian dualities and spacelike hypersurfaces in the lightcone," Mosc. Math. J. 9, 325-357 (2009).

${ }^{6}$ S. Izumiya, "Differential geometry from the viewpoint of Lagrangian or Legendrian singularity theory," in Singularity Theory, edited by D. Chéniot et al. (World Scientific, 2007), pp. 241-275.

${ }^{7}$ S. Izumiya, Y. Jiang, and D. H. Pei, "Lightcone dualities for curves in the sphere," Q. J. Math. Oxford Ser. 64, 221-234 (2013).

${ }^{8}$ J. J. Koenderink, Solid Shape (The MIT Press, 1990).

${ }^{9}$ H. L. Liu and S. D. Jung, "Hypersurfaces in lightlike cone," J. Geom. Phys. 58, 913-922 (2008).

${ }^{10}$ T. Nagai, "The Gauss map of a hypersurface in Euclidean sphere and the spherical Legendrian duality," Topol. Appl. 159, 545-554 (2012).

${ }^{11}$ I. R. Porteous, "Some remarks on duality in $S^{3}$," Geometry and Topology of Caustics, Banach Center Publications Vol. 50 (Polish Academy of Sciences, Warsaw, 2004), pp. 217-226.

${ }^{12}$ M. C. Romero Fuster, "Sphere stratifications and the Gauss map," Proc. - R. Soc. Edinburgh, Sect. A: Math. 95, 115-136 (1983).

${ }^{13}$ O. P. Shcherbak, "Projectively dual space curves and Legendre singularities," Sel. Math. Sov. 5, 391-421 (1986).

${ }^{14}$ F. Schwarz, "Asymptotic expansions of Fourier integrals with light cone singularities," J. Math. Phys. 13, 1621 (1972).

${ }^{15}$ F. Finster, "Light-cone expansion of the Dirac sea in the presence of chiral and scalar potentials," J. Math. Phys. 41, 6689 (2000). 